\title{
Cdk5 Phosphorylation of WAVE2 Regulates Oligodendrocyte Precursor Cell Migration through Nonreceptor Tyrosine Kinase Fyn
}

\author{
Yuki Miyamoto, ${ }^{1}$ Junji Yamauchi, ${ }^{1,2}$ and Akito Tanoue ${ }^{1}$ \\ ${ }^{1}$ Department of Pharmacology, National Research Institute for Child Health and Development, Okura, Setagaya, Tokyo 157-8535, Japan, and ${ }^{2}$ Department \\ of Biological Sciences, Tokyo Institute of Technology, Midori, Nagatsuta, Yokohama 226-8501, Japan
}

\begin{abstract}
Myelin formation of the CNS is a complex and dynamic process. Before the onset of myelination, oligodendrocytes (OLs), the myelinforming glia of the CNS, proliferate and migrate along axons. Little is known about the molecular mechanisms underlying the early myelination processes. Here, we show that platelet-derived growth factor (PDGF), the crucial physiological ligand in early OL development, controls the migration of oligodendrocyte precursor cells (OPCs) through cyclin-dependent kinase 5(Cdk5). PDGF stimulates Cdk5 activity in a time-dependent manner, whereas suppression of $\mathrm{Cdk} 5$ by the specific inhibitor roscovitine or by the retrovirus encoding short-hairpin RNA for Cdk5 impairs PDGF-dependent OPC migration. The activation of Cdk5 by PDGF is mediated by the phosphorylation of the nonreceptor tyrosine kinase, Fyn, whose inhibition reduces PDGF-dependent OPC migration. Furthermore, Cdk5 regulates PDGF-dependent OPC migration through the direct phosphorylation of WASP (Wiskott-Aldrich syndrome protein)-family verprolinhomologous protein 2 (WAVE2). Cdk5 phosphorylates WAVE2 at Ser-137 in vitro. Infection of the WAVE2 construct harboring the Ser-137-to-Ala reduces PDGF-dependent migration. Together, PDGF regulates OPC migration through an as-yet-unidentified signaling cascade coupling Fyn kinase to Cdk5 phosphorylation of WAVE2. These results provide new insights into both the role of Cdk5 in glial cells and the molecular mechanisms controlling the early developmental stage of OLs.
\end{abstract}

Key words: oligodendrocytes; migration; phosphorylation; Cdk5; WAVE2; CNS

\section{Introduction}

Oligodendrocyte precursor cells (OPCs) are generated in the ventricular zones of the vertebrate CNS. OPCs then migrate long distances to their final destinations, where they extend branched processes and form myelin sheaths that wrap target axons to facilitate rapid axonal conduction (Lee et al., 2000; Bhat, 2003). To migrate widely throughout the CNS, OPCs need to respond to various growth factors (Barres and Raff, 1999; Miller, 2002). In the adult brain, OPCs may also be guided to sites of injury, where they contribute to myelin repair (Blakemore and Keirstead, 1999; Zhang et al., 1999).

The extracellular soluble signals related to the processes of oligodendrocyte (OL) development are known to include platelet-derived growth factor (PDGF), basic fibroblast growth factor (bFGF), HGF (hepatocyte growth factor), IGF-I (insulin-

\footnotetext{
Received April 7, 2008; revised June 20, 2008; accepted July 11, 2008.

This work was supported by Grants-in-Aid for Scientific Research from both the Japanese Ministry of Education, Culture, Sports, Science, and Technology and the Japanese Ministry of Human Health and Welfare, by Research Fellowships from the Japan Society for the Promotion of Science for Young Scientists, and by research grants from the Astellas Metabolic Disease Foundation, the Human Science Foundation, the Kato Memorial Bioscience Foundation, and the Takeda Science Foundation. We thank Drs. E. M. Shooter, Y. Kaziro, and S. Hisanaga for insightful discussions. We thank R. Kimura for her participation in a helpful discussion and for providing encouragement.

Correspondence should be addressed to Junji Yamauchi, Department of Pharmacology, National Research Institute for Child Health and Development, 2-10-1 Okura, Setagaya, Tokyo 157-8535, Japan. E-mail: jyamauchi@nch.go.jp.

D0I:10.1523/JNEUROSCI.1482-08.2008

Copyright $\odot 2008$ Society for Neuroscience $\quad$ 0270-6474/08/288326-12\$15.00/0
}

like growth factor-I), GGFs (glial growth factors), and neurotrophic factors (Lee et al., 2000). Among these, PDGF-AA is one of the key regulators of early OL development. PDGF is synthesized by neurons and astrocytes and interacts with the cognate PDGF receptor $\alpha$ (PDGFR $\alpha$ ) on OPCs (Richardson et al., 1988; Yeh et al., 1991; Mudhar et al., 1993). PDGF binding to PDGFR $\alpha$ mediates OPC proliferation, migration, and differentiation in vitro (Noble et al., 1988; Richardson et al., 1988). These functions are supported by the observation that PDGF-A-deficient mice result in reduced numbers of mature OLs and hypomyelination (Fruttiger et al., 1999). Although many studies have demonstrated the importance of PDGF in OL development, our knowledge of how PDGF regulates OL development, that is, its precise mechanisms and its functions, remains largely fragmentary.

Cell migration is a hallmark of development. The migration of cells in response to growth factors is a complex process that requires the acquisition of spatial and temporal polarities between the front and the rear of the cells. Lamellipodia and filopodia at the leading fronts extend the cells toward stimuli and organize new adhesions with the substratum. These events require dynamic rearrangements of actin cytoskeletons. Cyclin-dependent kinase $5(\mathrm{Cdk} 5)$ is one such regulator. It belongs to the prolinedirected serine/threonine kinase family and is abundantly expressed in developing neuronal tissues. It is known that mice lacking Cdk5 exhibit a severely disrupted laminar structure of the cerebral cortex resulting from the inability of cortical neurons to 
migrate (Ohshima et al., 1996; Chae et al., 1997), but the role of Cdk5 in CNS glial cells has not been well investigated.

The Wiskott-Aldrich syndrome protein (WASP) family consists of the WASP subfamily (WASP and N-WASP) and the WASP-family verprolin-homologous protein (WAVE) subfamily (WAVE1, WAVE2, and WAVE3) (Takenawa and Miki, 2001). These proteins are composed of a basic region, a polyproline region, and a conserved verprolin homology, cofilin homology, and acidic domain (VCA) module. The VCA is the important module, because it is the module that functionally binds to actinrelated protein 2/3 (Arp2/3) and serves to scaffold monomeric actin. WASP family proteins are key regulators that link extracellular signals to actin reorganization. WASP and N-WASP specially bind to the Rho family small GTPase Cdc42 and control the formation of filopodia (Symons et al., 1996), whereas WAVE proteins constitutively regulate the formation of lamellipodia and membrane ruffling through the Rho family small GTPase Rac (Miki et al., 1998). WAVE1 knock-out mice exhibit severe deficits in learning and memory and prominent defects in CNS myelination (Dahl et al., 2003; Kim HJ et al., 2006). Mice lacking WAVE2 exhibit impaired vascular endothelial cell migration, which results in a cardiovascular development defect (Yamazaki et al., 2003). These observations in knock-out mice remind us of the relationship of WASP family proteins with OPC migration.

Because OPC migration precedes myelination, as noted above, it is worthwhile to identify the molecular mechanisms underlying the migration process. Thus, in the present study, we ask first whether $\mathrm{Cdk} 5$ participates in regulating OPC migration. We find that roscovitine, an inhibitor of Cdk5, dramatically inhibits PDGF-induced migration of primary OPCs. In addition, $\mathrm{Cdk} 5$ activity is increased by stimulation of OPCs with PDGF. We also demonstrate that the nonreceptor tyrosine kinase Fyn phosphorylates and activates Cdk5 after stimulation with PDGF. Furthermore, we demonstrate that Cdk5 regulates OPC migration through WAVE2 phosphorylation.

\section{Materials and Methods}

Materials. The following antibodies were purchased: anti-NG2 from Millipore Bioscience Research Reagents; anti- $\beta$-actin from BD Biosciences PharMingen; anti-Cdk5 (C-8) anti-p35 (C-19), anti-Fyn (FYN3), anti-WAVE2 (H-110) (for immunoblot), and anti-WAVE2 (C-14) (for immunoprecipitation) from Santa Cruz Biotechnology; antiphosphorylated (Ser) Cdk, anti-phosphorylated mitogen-activated protein kinase (MAPK)/Cdk, and anti-phosphorylated Cdc2 (Tyr15) from Cell Signaling Technology; anti-Fyn (clone 1S) and anti-phosphorylated tyrosine (4G10) from Millipore; anti-green fluorescent protein (GFP) from MBL; anti-ZsGreen from Clontech Takara Bio; and horseradish peroxidase-conjugated anti-mouse or anti-rabbit secondary IgGs from GE Healthcare Bio-Sciences. The Alexa Fluor 488 anti-mouse IgG and Alexa Fluor 546 anti-rabbit IgG were obtained from Invitrogen. Recombinant mouse PDGFR $\alpha$-Fc chimera was obtained from R\&D Systems. Roscovitine was purchased from Biomol. PP1 and PP3 were obtained from EMD Chemicals. Poly-L-lysine (PLL) was purchased from Sigma-Aldrich.

RNA preparation and reverse transcription-PCR analysis. Total RNA was prepared with Trizol (Invitrogen) reagent. The cDNAs were prepared from $1 \mu \mathrm{g}$ of total RNA with the Superscript III enzyme (Invitrogen), according to the manufacturer's instructions. PCR amplification was performed for the indicated number of cycles, each cycle consisting of denaturation at $94^{\circ} \mathrm{C}$ for $1 \mathrm{~min}$, annealing at $59-62^{\circ} \mathrm{C}$ (depending on $\mathrm{Tm}$ values of primer pairs) for $1 \mathrm{~min}$, and extension at $72^{\circ} \mathrm{C}$ for $1 \mathrm{~min}$ at $40 \mathrm{cy}$ cles. The primers used were as follows: Fyn, $5^{\prime}$-ATGGGCTGTGTGCAATGTAAGGATAAAG-3' (sense) and 5' -TCACAGGTTTTCACCGGGGC-3' (antisense); Src, 5'-ATGGGCAGCAACAAGAGCAAGCC-3'(sense) and 5'-CTATAGGTTCTCCCCGGGCTG-3' (antisense); Lyn, 5' -ATGGGAT-
GTATTAAATCAAAAAGGAAAGACAATCTGAATG-3' (sense) and 5'CTATGGCTGCTGCTGATACTGC-3' (antisense); Yes, 5' -ATGGGCTGCATTAAAAGTAAAGAAAAC-3' (sense) and 5' -TTATAAATTTTCTCCTGGTTGGTACTG-3' (antisense); WAVE1, 5'-ATGCCGCTGGTGAAAAGAAACATCGAC-3' (sense) and 5'-CATCTGACTGAATGGCAAGGCAG-3' (antisense); WAVE2, 5' -ATGCCCTTAGTAACCAGGAACATCGAG-3' (sense) and 5'-GGAGGCAGAGTCCGACTG-3' (antisense); WAVE3, 5'-ATGCCTTTAGTGAAGAGGAACATTGAACC-3' (sense) and $5^{\prime}$-CGGCTGTACTTGCAAAGCGTG-3' (antisense); $\beta$-actin, $5^{\prime}$-ATGGATGACGATATCGCTGCGCTC-3' (sense) and 5'-CTAGAAGCATTTGCGGTGCACGATG-3' (antisense).

Plasmids. The region encoding rat WAVE2 protein was amplified by reverse transcription (RT)-PCR from rat OL total RNA and ligated into the mammalian pRetroX-IRES-ZsGreen1 vector (Clontech Takara Bio) or pLEGFP-C1 vector (Clontech Takara Bio). The construct of WAVE2 harboring the Ser-137-to-Ala mutation or Ser-137-to-Glu mutation was created from pRetroX-IRES-ZsGreen1-WAVE2 or pLEGFP-C1WAVE2 by Pfu-based PCR using the QuikChange Site-Directed $\mathrm{Mu}$ tagenesis Kit (Stratagene). The WAVE2 fragment cDNAs, SH (amino acids 1-170), proline (amino acids 171-284), and VCA (amino acids 285-498), were inserted into pLEGFP-C1. All sequences were confirmed by $\mathrm{ABI}$ automatic sequencers.

The plasmid encoding sequences for short-hairpin RNAs. The oligonucleotides used were as follows: Cdk5 (starting from nucleotide 531 of rat Cdk5) sense oligonucleotide, 5'-GATCCGCTGTACTCCACGTCCATCTTCAAGAGAGATGGACGTGGAGTACAGCTTTTTTACGCGTG-3'; Cdk5 antisense oligonucleotide, 5'-AATTCACGCGTAAAAAAGCTGTACTCCACGTCCATCTCTCTTGAAGATGGACGTGGAGTACAGCG-3'; p35 (starting from nucleotide 811 of rat p35) sense oligonucleotide, 5'-GATCCGATGCTGCAGATCAATGCTTTCAAGAGAAGCATTGATCTGCAGCATCTTTTTTACGCGTG-3'; p35 antisense oligonucleotide, 5'-AATTCACGCGTAAAAAAGATGCTGCAGATCAATGCTTCTCTTGAAAGCATTGATCTGCAGCATCG-3'; p39 (starting from nucleotide 705 of human/mouse/rat p39) sense oligonucleotide, 5' -GATCCCCTGGTGTTCGTGTACCTGTTCAAGAGACAGGTACACGAACACCAGGTTTTTTACGCGTG-3'; p39 antisense oligonucleotide, 5'-AATTCACGCGTAAAAAACCTGGTGTTCGTGTACCTGTCTCTTGAACAGGTACACGAACACCAGG-3'; Fyn (starting from nucleotide 283 of rat Fyn) sense oligonucleotide, 5' -GATCCGCACGGACAGAAGATGACCTTCAAGAGAGGTCATCTTCTGTCCGTGCTTTTTTACGCGTG-3'; Fyn antisense oligonucleotide, 5' -AATTCACGCGTAAAAAAGCACGGACAGAAGATGACCTCTCTTGAATGGTAAGTGTGGTTTCCAGCG-3'; Yes (starting from nucleotide 263 of mouse/rat Yes) sense oligonucleotide, 5'-GATCCGCAGGTGGTGTCACTATATTTTCAAGAGAAATATAGTGACACCACCTGCTTTTTTACGCGTG-3'; Yes antisense oligonucleotide, 5'-AATTCACGCGTAAAAAAGCAGGTGGTGTCACTATATTTCTCTTGAAAATATAGTGACACCACCTGCG-3'; WAVE1 (starting from nucleotide 492 of human/mouse/rat WAVE1) sense oligonucleotide, 5' -GATCCGATGTTGCAAGATACAGAGTTCAAGAGACTCTGTATCTTGCAACATCTTTTTTACGCGTG-3'; WAVE1 antisense oligonucleotide, 5'-AATTCACGCGTAAAAAAGATGTTGCAAGATACAGAGTCTCTTGAACTCTGTATCTTGCAACATCG-3'; WAVE2 (starting from nucleotide 265 of human/ rat WAVE2) sense oligonucleotide, 5'-GATCCGTGTCACTGCAAGGAATCATTCAAGAGATGATTCCTTGCAGTGACACTTTTTTACGCGTG-3'; WAVE2 antisense oligonucleotide, 5'-AATTCACGCGTAAAAAAGTGTCACTGCAAGGAATCATCTCTTGAATGATTCCTTGCAGTGACACG-3'; WAVE3 (starting from nucleotide 180 of human/rat WAVE3) sense oligonucleotide, 5'-GATCCGCAACTTCTACATCAGAGCATTCAAGAGATGCTCTGATGTAGAAGTTGCTTTTTTACGCGTG-3'; WAVE3 antisense oligonucleotide, 5'-AATTCACGCGTAAAAAAGCAACTTCTACATCAGAGCATCTCTTGAATGCTCTGATGTAGAAGTTGCG-3'; luciferase sense oligonucleotide, 5'-GATCCGGCCATTCTATCCTCTAGAGTTCAAGAGACTCTAGAGGATAGAATGGCCTTTTTTAGATCTC-3'; and luciferase antisense oligonucleotide, 5' ${ }^{\prime}$-AATTCAGATCTAAAAAAGGCCATTCTATCCTCTAGAGTCTCTTGAACTCTAGAGGATAGAATGGCCG-3'. These nucleotide segments do not have any significant homology to mammalian gene sequences. The sense oligonucleotides for Cdk5, p35, p39, Fyn, Yes, WAVE1, WAVE2, WAVE3, and luciferase were each an- 
nealed with their corresponding antisense oligonucleotides. The annealed duplexes were ligated into the pSIREN-RetroQ-ZsGreen plasmid.

Cell culture. OPCs were isolated from day 16 embryos of Sprague Dawley rats, as previously described (Miyamoto et al., 2007). Briefly, cerebral cortices were dissected, dissociated with $0.25 \%$ trypsin (Invitrogen), triturated, and passed through $70 \mu \mathrm{m}$ pore-size meshes. Cells were then collected, resuspended in MEM containing $10 \%$ FBS, $50 \mathrm{U} / \mathrm{ml}$ penicillin, and $50 \mu \mathrm{g} / \mathrm{ml}$ streptomycin, and seeded on PLL-coated dishes. After two such passages, the cells were cultured on noncoated Petri dishes (Barloworld Scientific). On the second day of culture, the medium was changed to DMEM-based serum-free medium with N-2 supplement (Invitrogen), $100 \mu \mathrm{g} / \mathrm{ml} \mathrm{BSA} \mathrm{(Sigma-Aldrich),} 60 \mu \mathrm{g} / \mathrm{ml} N$-acetyl-Lcysteine (Sigma-Aldrich), $1 \mathrm{ng} / \mathrm{ml}$ forskolin (Sigma-Aldrich), $5 \mathrm{ng} / \mathrm{ml}$ neurotrophin-3 (Peprotech), $10 \mathrm{ng} / \mathrm{ml}$ bFGF (Peprotech), and $10 \mathrm{ng} / \mathrm{ml}$ PDGF-AA (Peprotech). The cells were cultured for an additional $2 \mathrm{~d}$, then used as OPCs. Human epithelial 293 T cells were cultured in DMEM containing $10 \% \mathrm{FBS}, 50 \mathrm{U} / \mathrm{ml}$ penicillin, and $50 \mu \mathrm{g} / \mathrm{ml}$ streptomycin. OPCs were pretreated with PP1 $(10 \mu \mathrm{M}, 1 \mathrm{~h}), \mathrm{PP} 3(10 \mu \mathrm{M}, 1 \mathrm{~h})$, or roscovitine $(10 \mu \mathrm{M}, 1 \mathrm{~h})$ before stimulation with PDGF $(10 \mathrm{ng} / \mathrm{ml}$, 0-1440 min). To confirm cell viability under these experimental conditions, OPCs and 293T cells were stained with $0.4 \%$ trypan blue. Trypan blue-incorporating cells numbered $<2 \%$ in each experiment.

Transfection. The retroviral expression vectors and the envelope expression vector pVSV-G were cotransfected into GP2-293 cells with the Calcium Phosphate Transfection Kit (Clontech Takara Bio), as previously described (Miyamoto et al., 2007). Briefly, at $2 \mathrm{~d}$ after transfection, the culture supernatants were centrifuged at $10,000 \mathrm{rpm}$ for $8 \mathrm{~h}$ to concentrate the recombinant viruses. The virus pellets were suspended in culture medium and used to infect purified OPCs. At $1 \mathrm{~d}$ after infection, OPCs were used in migration assays. Plasmid DNAs were transfected into 293T cells by the Calcium Phosphate Transfection Kit.

Migration assay using reaggregated OPCs. To mimic physiological conditions, OPC migration was measured using reaggregated OPCs (Yamauchi et al., 2004). The retrovirus-infected or noninfected OPC reaggregates were formed by plating OPCs on Ultra Low Attachment dishes (Corning) for 5-6 h. The reaggregated OPCs were then plated onto PLL-coated dishes in the presence or absence of $10 \mathrm{ng} / \mathrm{ml}$ PDGF with 10 $\mu \mathrm{M}$ AraC. After incubation at $37^{\circ} \mathrm{C}$ for $16 \mathrm{~h}$, OPCs were fixed with $4 \%$ paraformaldehyde, blocked, and immunostained. The images were captured with a TE-300 fluorescence microscope system (Nikon) or a DMI4000B fluorescence microscope system (Leica). The distance of migration was calculated by measuring the change in size of the reaggregates over time, subtracting the average initial size of the reaggregates, and dividing the remaining distance in half. More than five aggregates per dish were measured in two independent experiments.

Boyden chamber migration assay. Cell migration was routinely measured using a 24-well Boyden chamber (BD Biosciences), as previously described (Yamauchi et al., 2004). Polyethylene terephthalate $(8 \mu \mathrm{m}$ pore size) filters were coated with PLL. OPCs $\left(2 \times 10^{5}\right.$ cells for noninfected cells; $1 \times 10^{6}$ cells for infected cells) in $500 \mu$ l of media per well were loaded into the upper chambers, which were inserted into the tissue culture wells, each of which contained $500 \mu \mathrm{l}$ of media either with or without $10 \mathrm{ng} / \mathrm{ml}$ PDGF and $10 \mu \mathrm{M}$ AraC per well. After incubation at $37^{\circ} \mathrm{C}$ for $16 \mathrm{~h}$, the filters were stained with Giemsa solution or fixed with paraformaldehyde to detect cells expressing GFP. The number of migrating cells, either stained or GFP-fluorescent, at the bottom surface of the filters was counted at four fields per filter in two independent experiments.

Immunofluorescence. OPCs were fixed in $4 \%$ paraformaldehyde in PBS for $8 \mathrm{~min}$ at room temperature. The fixed cells were permeabilized and blocked in PBS containing 20\% normal goat serum and 0.1\% Tween 20 for $1 \mathrm{~h}$ at room temperature. Blocked cells were incubated with primary antibodies overnight at $4^{\circ} \mathrm{C}$. Unbound primary antibodies were removed by washing three times with PBS containing $0.05 \%$ Tween 20 . Washed cells were then incubated with secondary antibodies at room temperature for $1 \mathrm{~h}$. After three rinses with PBS containing $0.05 \%$ Tween 20, cells were mounted on a Vectashield (Vector Laboratories) and observed under a microscope.

Immunoprecipitation and immunoblotting. Cells were lysed in lysis buffer (50 mм HEPES-NaOH, pH 7.5, 20 mм MgCl 2,150 mm NaCl, 1 mм dithiothreitol, $1 \mathrm{~mm}$ phenylmethane sulfonylfluoride, $1 \mu \mathrm{g} / \mathrm{ml}$ leupeptin, $1 \mathrm{~mm}$ EDTA, $1 \mathrm{~mm} \mathrm{Na} \mathrm{VO}_{4}, 10 \mathrm{~mm} \mathrm{NaF}$, and $0.5 \% \mathrm{NP}-40$ ) and centrifuged as previously described (Miyamoto et al., 2006). Aliquots of the supernatants were mixed with a protein $\mathrm{G}$ resin preabsorbed with various antibodies. The immunoprecipitates or the proteins in the cell lysates were denatured and then separated on SDS-polyacrylamide gels. The electrophoretically separated proteins were transferred to polyvinylidene difluoride membranes, blocked, and immunoblotted. The bound antibodies were detected using the ECL system (GE Healthcare). Captured band images were analyzed with ImageJ software (available from http:// rsb.info.nih.gov/ij/). At least three separate experiments were performed, and a representative experiment is shown in the figures.

Cdk5 kinase assay. Immunoprecipitated Cdk5 was incubated with 100 $\mathrm{ng} / \mu \mathrm{l}$ histone $\mathrm{H} 1$ as the substrate in $30 \mu \mathrm{l}$ of reaction buffer $(20 \mathrm{~mm}$ HEPES-NaOH, pH 7.5, 1 mm dithiothreitol, $10 \mathrm{~mm} \mathrm{NaF,} 0.1 \mathrm{~mm}$ phenylmethane sulfonylfluoride, $0.1 \mu \mathrm{g} / \mathrm{ml}$ leupeptin, and $0.1 \mathrm{~mm}$ EDTA) containing $20 \mu \mathrm{M} \mathrm{ATP}$ at $30^{\circ} \mathrm{C}$ for $30 \mathrm{~min}$ and then chilled on ice. The supernatants were collected and immunoblotted with an antiphosphorylated MAPK/Cdk antibody. At least three separate experiments were performed. The band intensity was also quantified. A representative experiment is shown in the figure.

In vitro WAVE2 phosphorylation reaction. Immobilized WAVE2 proteins were incubated with $100 \mathrm{ng} / \mu \mathrm{l}$ recombinant Cdk5/p25 protein (Millipore) in $30 \mu \mathrm{l}$ of reaction buffer containing $20 \mu \mathrm{M} \mathrm{ATP}$ at $30^{\circ} \mathrm{C}$ for $15 \mathrm{~min}$ and then chilled on ice. The immobilized phosphorylated WAVE2 was washed with reaction buffer and immunoblotted with an anti-phosphorylated Cdk antibody. At least three separate experiments were performed. The band intensity was also quantified. A representative experiment is shown in the figure.

Statistical analysis. Values shown represent the mean \pm SD from separate experiments. Student's $t$ test was performed for intergroup comparisons $\left({ }^{*} p<0.01\right)$.

\section{Results}

\section{Cdk5 mediates PDGF-induced migration of oligodendrocyte precursor cells}

OPCs arise from the ventricular zone, which in an earlier developmental stage generates neuronal precursors. OPCs then migrate dorsally and ventrally, and finally differentiate into OLs (Lee et al., 2000; Bhat, 2003). OPC migration is known to be regulated by many growth factors secreted from neurons (Barres and Raff, 1999; Miller, 2002). In an attempt to study whether these factors are involved in OPC migration, we first used conditioned media from cortical neurons and measured OPC migration from the reaggregates. Figure $1, A$ and $B$, illustrates that conditioned media from cortical neurons stimulated OPC migration so that it increased $\sim 2.5$-fold, suggesting that relevant growth factors may be contained in the conditioned media.

We suspected that one of these growth factors was PDGF. In the CNS, PDGF is known to regulate many aspects of OL development and to maintain OPCs in a bipolar migratory state. Active PDGF is composed of homodimers or heterodimers of the PDGF-A or -B chain. PDGF-A is synthesized by neurons as well as by astrocytes, whereas PDGF-B is produced by capillary endothelial cells. PDGF binds and activates two cognate high-affinity cell-surface receptors, namely, PDGFR $\alpha$ and PDGFR $\beta$. (Heldin and Westermark, 1989). OPCs mainly express PDGFR $\alpha$, which is activated by all three dimeric isoforms of PDGF (AA, AB, and $\mathrm{BB}$ ) (Pringle et al., 1989). To determine whether PDGF in the conditioned media from cortical neurons is actually the factor that controls OPC migration, we added the PDGF scavenger PDGFR $\alpha$-Fc to the conditioned media. Removal of PDGF with PDGFR $\alpha$-Fc resulted in the inhibition of OPC migration by $\sim 40 \%$ (Fig. $1 A, B$ ), suggesting that PDGF is indeed a factor that regulates OPC migration under our experimental conditions. 


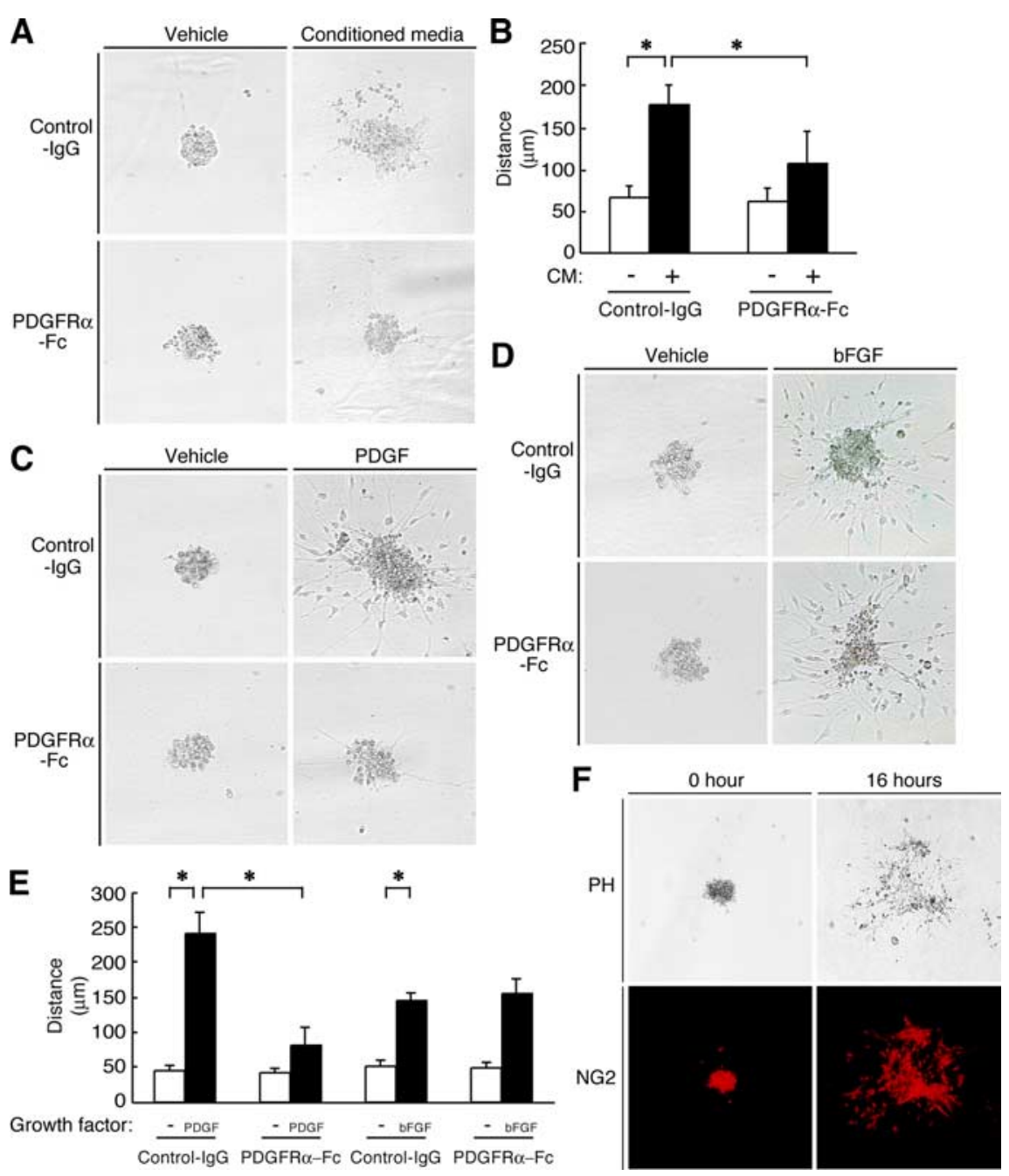

Figure 1. PDGF promotes migration from OPC aggregates. $A, B, O P C$ reaggregates were placed onto PLL-coated dishes and incubated with normal medium or conditioned medium (CM) from cortical neurons containing $2 \mu \mathrm{g} / \mathrm{ml}$ control lgG or PDGFR $\alpha$-Fc and $10 \mu \mathrm{m} \mathrm{AraC}$. After $16 \mathrm{~h}, \mathrm{OPC}$ reaggregates were fixed and observed under phase-contrast microscopy. The distance of migration was measured. $\boldsymbol{C}-\boldsymbol{E}$, In the presence of control lgG or PDGFR $\alpha-\mathrm{Fc}, 0 \mathrm{PC}$ aggregates were incubated with or without 10 $\mathrm{ng} / \mathrm{mI}$ PDGF-AA or bFGF in the presence of $10 \mu \mathrm{m} \mathrm{AraC}$. After $16 \mathrm{~h}$, reaggregates were fixed and observed under phase-contrast microscopy. Representative images of cells are shown. The distance of migration was measured. $\boldsymbol{F}, 0 \mathrm{PC}$ reaggregates were stained with an antibody against NG2 (red) before or after $16 \mathrm{~h}$ of incubation with $10 \mathrm{ng} / \mathrm{ml} \mathrm{PDGF}$. The representative phase-contrast images (PH) and fluorescence images (NG2) are shown. Data were evaluated using Student's $t$ test $\left({ }^{*} p<0.01\right)$.

(Fig. $1 F$ ). Together, these results suggest that endogenous PDGF can promote migration of OPCs.

Because Cdk5 plays a critical part in neuronal migration (Ohshima et al., 1996; Chae et al., 1997), we asked whether Cdk5 is involved in PDGF-induced OPC migration as well. In migration assays using OPC reaggregates, roscovitine, an inhibitor of Cdk5, significantly reduced PDGFinduced migration (Fig. 2A,B). To further examine the role of $\mathrm{Cdk} 5$ in PDGFinduced OPC migration, we performed migration assays using a Boyden chamber. OPCs were plated on the filters in the upper wells and allowed to migrate out into the lower chambers through $8 \mu \mathrm{m}$ pores. PDGF was placed in the lower wells of the Boyden chambers, forming a concentration gradient that extended into the upper wells. As shown in Figure 2, $C$ and D, stimulation with PDGF promoted OPC migration $\sim 2.0$-fold. This effect was blocked by roscovitine (Fig. 2C,D). This outcome is consistent with the results from our reaggregate migration assay.

We next infected OPCs with a retrovirus expressing both GFP and a shorthairpin RNA (shRNA) sequence targeting Cdk5 or its activator p35 (Tsai et al., 1994). The expression of Cdk5 or p35 was specifically downregulated by infection with each shRNA, whereas expression of control proteins was unaffected (Fig. 2E). Knockdown of Cdk5 blocked PDGFinduced migration from OPC reaggregates (Fig. $2 F, G$ ). Consistently with this result, infection of an shRNA for Cdk5 also blocked OPC migration in Boyden chambers (Fig. $2 H, I$ ). However, knockdown of p35 had less of an inhibitory effect on PDGF-induced migration (Fig. 2 J, K).p39 is another Cdk5 regulator isolated from a hippocampus library (Tang et al., 1995).

To confirm that PDGF induces OPC migration, we performed migration assays using the reaggregates with recombinant PDGFAA. As shown in Figure 1, $C$ and $E$, PDGF increased OPC migration $\sim 5.0$-fold. To avoid any effects of PDGF on OPC proliferation, $\operatorname{AraC}(10 \mu \mathrm{M})$ was simultaneously added into the normal medium during the migration assay using PDGF. Under these conditions, we observed that $<2 \%$ of cells incorporated trypan blue. The effect of the addition of PDGF on OPC migration was much greater than that of the addition of conditioned media. This observation might be attributable to the presence of numerous factors in conditioned media, each of which may influence migration positively or negatively.

We next checked whether PDGFR $\alpha$-Fc is specific for the PDGF activity. As shown in Figure $1 C-E$, PDGFR $\alpha$-Fc specifically inhibited PDGF-induced migration, but did not inhibit migration induced by bFGF, another growth factor known to stimulate OPC migration (Mckinnon et al., 1990). In addition, most of the cells in reaggregates were positively stained with an antibody against NG2, which recognizes bipolar migratory OPCs p39-deficient mice do not exhibit detectable abnormalities in neuronal positioning in the nervous system, whereas p35 and p39 double knock-out mice display phenotypes that are identical to those of Cdk5 knock-out mice in terms of their neuronal compartments (Ko et al., 2001). Thus, to further elucidate whether p39 is involved in PDGF-induced migration, OPCs were infected with an shRNA for p39. Expression of p39 was specifically downregulated by infection with its shRNA, whereas expression of control proteins was unaffected (Fig. $2 N$ ). Knockdown of p39 partially inhibited PDGF-induced OPC migration in the Boyden chamber (Fig. $2 L, M$ ), implying that p39 is one candidate for Cdk5 activator in PDGF-dependent OPC migration. Because the inhibitory effect of the p39 shRNA was only partial, there might be one or more different primary regulator(s) in the signaling pathway controlling OPC migration by PDGF. Thus, these results hint at the existence of another possible activator of Cdk5 in OPC migration and provide the rationale and the impetus for subsequent experiments.

Furthermore, to clarify whether Cdk5 is activated by PDGF in 

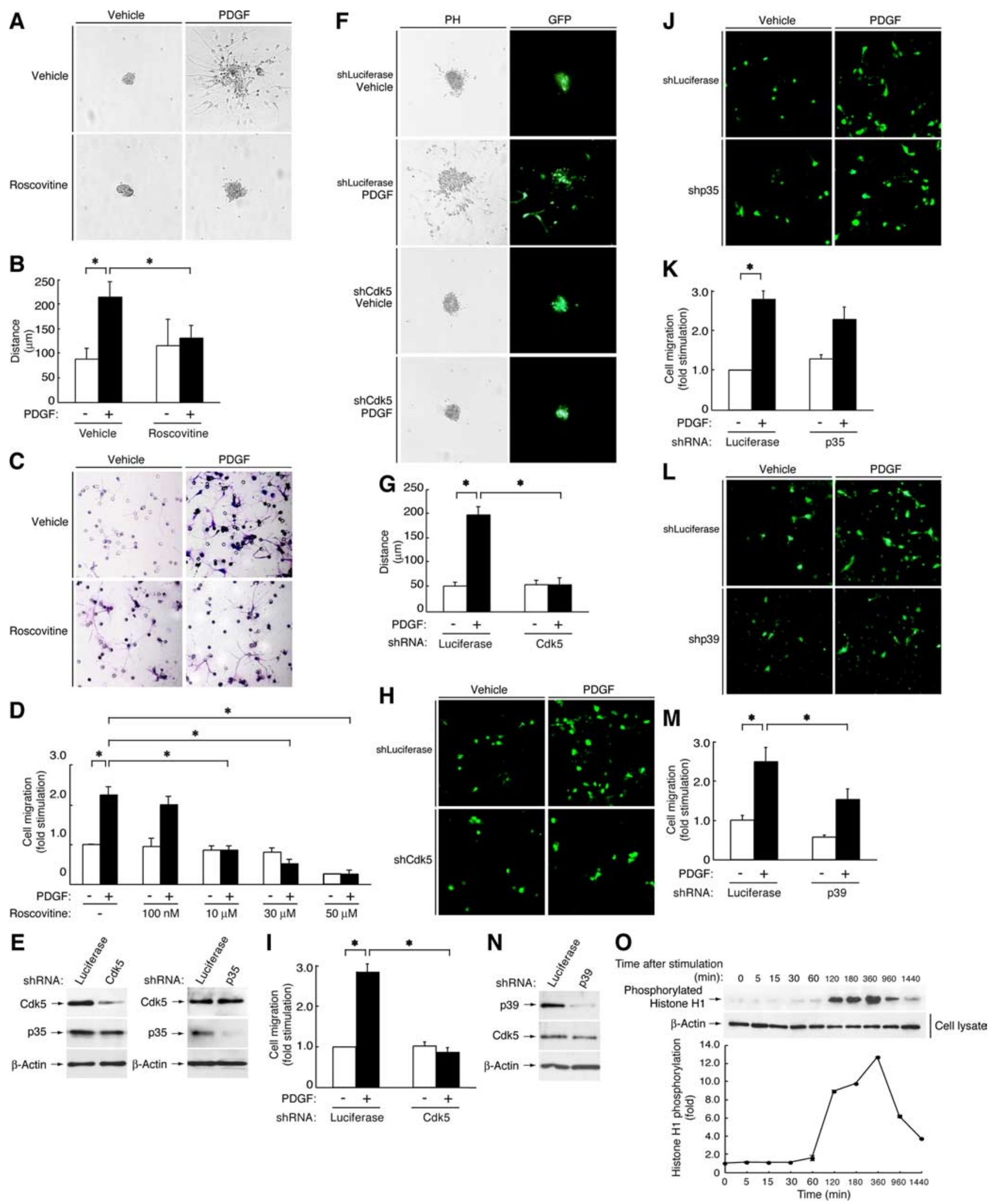

Figure 2. PDGF promotes $\mathrm{OPC}$ migration through $\mathrm{Cdk} 5 . A, B, O P C$ reaggregates were pretreated in the presence or absence of $10 \mu \mathrm{m}$ roscovitine and then incubated with or without PDGF. The distance of migration was measured. C, D, The migration of OPCs was measured using Boyden chambers. Filters were coated with PLL. OPCs were pretreated in the presence or absence of $100 \mathrm{~nm}$ (D), $10 \mu \mathrm{m}(\boldsymbol{C}, \boldsymbol{D}), 30 \mu \mathrm{m}(\boldsymbol{D})$, or $50 \mu \mathrm{m}(\boldsymbol{D})$ roscovitine and then incubated with or without PDGF. After 16h, OPCs were stained with Giemsa solution, and the number of migrating cells was counted. $\boldsymbol{E}$, To confirm the effects of shRNAs, the lysates from the infected OPCs were immunoblotted with an antibody against Cdk5, p35, or $\beta$-actin. F, G, OPCs were infected with Cdk5 or luciferase shRNA retroviral vector. At $24 \mathrm{~h}$ after infection, OPC reaggregates were formed, placed onto a PLL-coated dish, and incubated with or without PDGF for $16 \mathrm{~h}$. The representative phase-contrast (PH) and fluorescence images of GFP-positive reaggregates are shown. The distance of migration was measured. $\boldsymbol{H}-\boldsymbol{K}, \mathrm{OPC}$ were infected with $\mathrm{Cdk5}$, p35, or luciferase shRNA retrovirus with or without PDGF in Boyden chambers. The number of migrating cells was counted. $L, M, O P C s$ were infected with p39 or luciferase shRNA retrovirus with or without PDGF in Boyden (Figure legend continues.) 
OPCs, we measured the activity of Cdk5 using histone $\mathrm{H} 1$ as the substrate (Miyamoto et al., 2007). Histone H1 was phosphorylated in a time-dependent manner after PDGF stimulation (Fig. 2O). The level of phosphorylated histone $\mathrm{H} 1$ reached its maximum at $360 \mathrm{~min}$, when it had increased $\sim 13.0$-fold (Fig. 2O). Afterward, the Cdk5 activity gradually decreased, but could still be stimulated even after $24 \mathrm{~h}$ (Fig. 2O), indicating the key role of Cdk5 activation in OPC migration.

\section{Fyn kinase acts upstream of Cdk5 in PDGF-induced migration of oligodendrocyte precursor cells}

Various extracellular signals initiate signal transduction cascades by activating the cytoplasmic Src family of nonreceptor tyrosine kinases. Src family tyrosine kinases are important regulators of many cellular events such as proliferation, adhesion, migration, and differentiation (Thomas and Brugge, 1997). The Src family is composed of nine members, and some of its kinases, namely, Src, Fyn, Lyn, and Yes, are expressed in the brain (Thomas and Brugge, 1997). Among them, Fyn kinase is the only Src family tyrosine kinase that is upregulated during OL differentiation (Osterhout et al., 1999). Fyn kinase is also known to be the regulator of myelination in the CNS (Umemori et al., 1994). Thus, it is conceivable that Fyn kinase may also regulate OPC migration preceding myelination. To find out, we treated OPCs with PP1, the specific inhibitor of Src family tyrosine kinases. Figure $3 \mathrm{~A}$ shows that PP1 abolished PDGF-induced OPC migration. On the other hand, PP3, a PP1 analog that is incapable of inhibiting Src family tyrosine kinases, had no effect on PDGF-induced migration (Fig. 3A).

To compare the expression levels of the various Src family tyrosine kinases in OPCs, we analyzed the mRNA levels of Fyn, Src, Lyn, and Yes by RT-PCR. As we expected, a large amount of Fyn was expressed in OPCs, and small amount of Yes was expressed (Fig. 3B). Lyn has been reported to be expressed in OPCs (Osterhout et al., 1999), but we failed to detect any expression of Lyn under our experimental conditions. Next, to clarify which Src-type nonreceptor tyrosine kinase is involved in PDGFinduced OPC migration, we infected our OPCs with an shRNA specifically targeting Fyn or Yes. Expression of Fyn or Yes was specifically downregulated by infection with its respective shRNA, whereas expression of control mRNAs was unaffected (Fig. 3C). Knockdown of Fyn inhibited PDGF-induced migration in the Boyden chamber (Fig. $3 D, E$ ) as well as from reaggregates (Fig. $3 F, G$ ). In contrast, infection with an shRNA for Yes did not have a significant effect on migration (Fig. $3 H$ ), suggesting that PDGF regulates OPC migration primarily through Fyn.

To examine whether PDGF stimulates the intrinsic activity of Fyn kinase, we measured autophosphorylation of Fyn kinase. OPCs were lysed, immunoprecipitated with an anti-Fyn antibody, and immunoblotted with an anti-phospho-tyrosine antibody. Fyn kinase was autophosphorylated in response to PDGF stimulation (Fig. 3I). The autophosphorylation levels of Fyn kinase began to increase at $30 \mathrm{~min}$ and reached a maximum at 360 min, having increased $\sim 7.0$-fold (Fig. $3 I$ ). The tyrosine phosphorylation of Fyn kinase was observed even after $24 \mathrm{~h}$. Therefore, PDGF can stimulate Fyn kinase to promote OPC migration.
It has been reported that Fyn kinase phosphorylates Cdk5 at Tyr-15 to mediate semaphorin 3A-induced growth-cone collapse (Sasaki et al., 2002). Thus, we tested whether Fyn kinase phosphorylates Cdk5 after PDGF stimulation in OPCs. As shown in Figure $4 A$, Cdk5 that was phosphorylated by Fyn kinase at Tyr-15 was observed in a time-dependent manner. The level of tyrosine phosphorylation reached a maximum at $360 \mathrm{~min}$ after the addition of PDGF, having increased $\sim 9.0$-fold (Fig. $4 A$ ). It remained activated for at least $24 \mathrm{~h}$ (Fig. $4 A$ ). We next examined whether Cdk5 phosphorylation at Tyr-15 is mediated by Fyn kinase. Treatment of OPCs with PP1 reduced both PDGF-induced phosphorylation of Cdk5 at Tyr-15 (Fig. $4 B$ ) and the activation of Cdk5 (Fig. 4C). Furthermore, to confirm the involvement of Fyn kinase in the phosphorylation and activation of Cdk5, we infected OPCs with an shRNA for Fyn. Consistently with the results of PP1 treatment, Fyn knockdown inhibited both Cdk5 phosphorylation at Tyr-15 (Fig. 4D) and activation (Fig. 4E) of Cdk5 induced by PDGF stimulation. These results indicate that PDGF promotes migration through a signaling pathway that depends on Fyn kinase, which is upstream of Cdk5.

\section{WAVE2 is essential for oligodendrocyte precursor cell migration regulated by PDGF}

When cells migrate, filopodia and lamellipodia are formed at their leading edge. Filopodia and lamellipodia are made up of actin filaments and are formed mainly through actin polymerization. WAVE proteins are key regulators of this actin polymerization via their direct interaction with the Arp2/3 complex. To date, three WAVE proteins have been characterized (Takenawa and Miki, 2001). Because OPC migration involves dynamic morphological changes driven by actin cytoskeletal rearrangements, it is possible that WAVE proteins are also involved in PDGF-induced OPC migration. We first examined the expression profile of WAVE family proteins in OPCs. As shown in Figure $5 A$, all WAVE mRNAs, namely, WAVE1, WAVE2, and WAVE3, were detected in OPCs using RT-PCR.

Next, to clarify which WAVE protein or proteins participate in OPC migration, we infected OPCs with an shRNA targeting WAVE1, WAVE2, or WAVE3, to knock down expression of each endogenous protein. The expression of each WAVE mRNA was specifically downregulated by infection with its respective shRNA, whereas the expression of control molecules was unaffected, as detected by RT-PCR (Fig. 5B). Using these specific shRNA constructs, we tested the effects on PDGF-induced OPC migration. Knockdown of WAVE2 completely blocked PDGFinduced OPC migration in Boyden chambers (Fig. 5C,D) as well as from reaggregated OPCs (Fig. $5 E, F$ ). Knockdown of WAVE1 decreased migration in Boyden chambers by $\sim 40 \%$ (Fig. $5 C, D$ ). Infection with the WAVE3 shRNA, on the other hand, did not have an obvious effect on migration (Fig. 5C,D). Because, as this shows, WAVE2 plays a primary role in OPC migration, we focused on WAVE2 and proceeded with our experiments.

\section{Cdk5 phosphorylates Ser-137 of WAVE2}

Biochemical and bioinformatics data show that WAVE2 possesses various phosphorylation sites of kinases responsible for the 

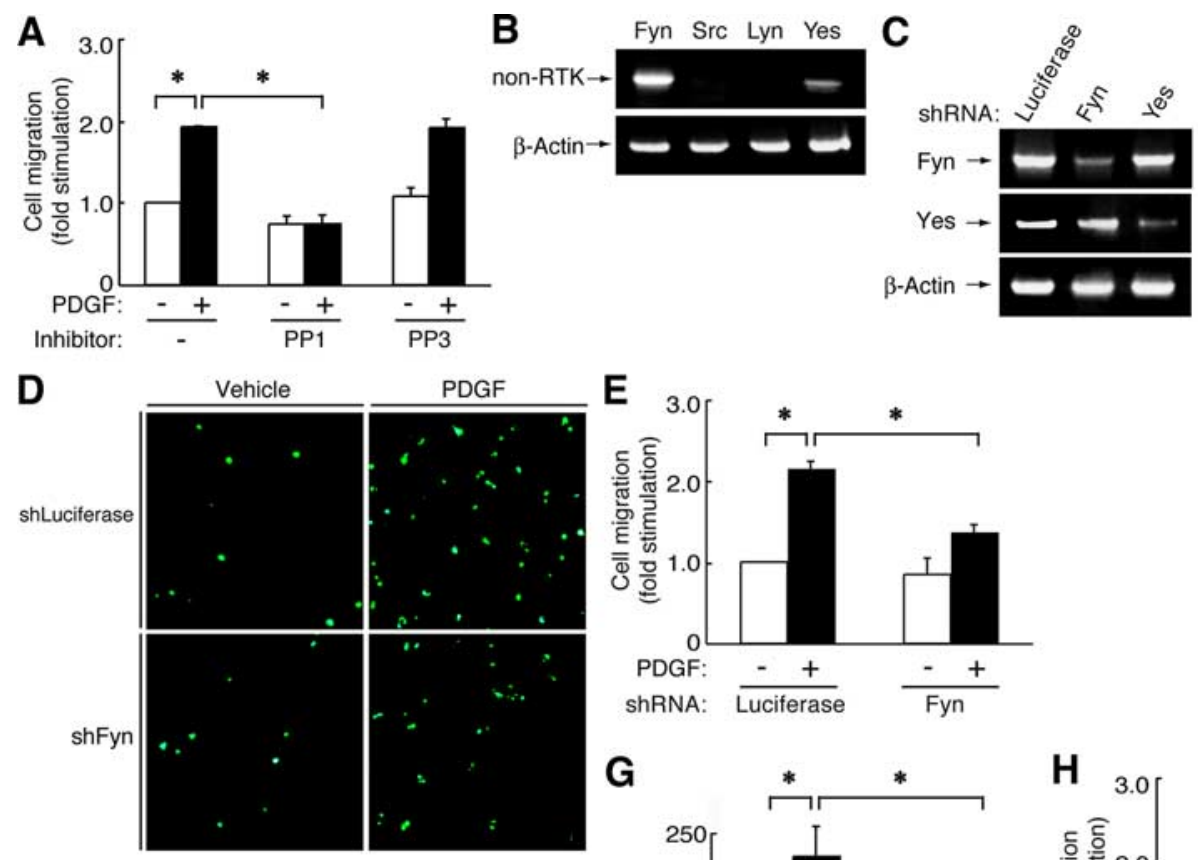

$\mathbf{F}$

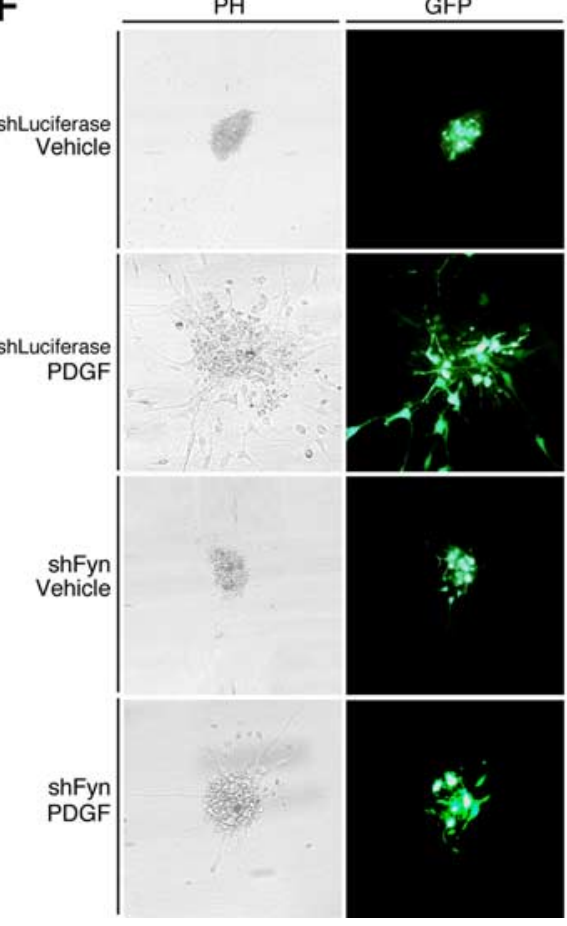

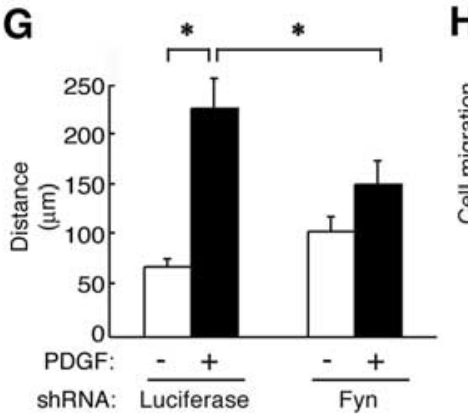

G

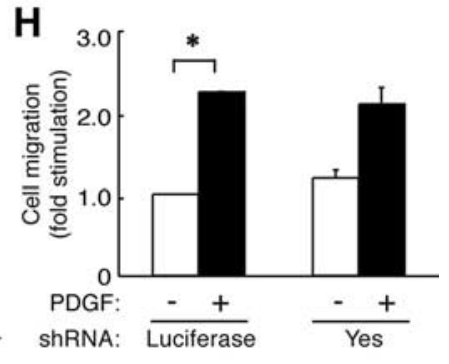

| Time after stimulation

Phosphorylated

$$
\text { (min): } \begin{array}{lllllllllll}
0 & 5 & 15 & 30 & 60 & 120 & 180 & 360 & 960 & 1440
\end{array}
$$
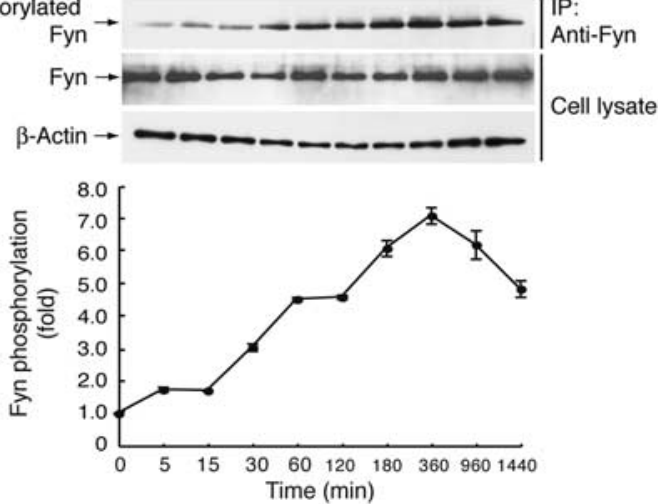

Figure 3. PDGF promotes $\mathrm{OPC}$ migration through Fyn kinase. $A, 0 P C s$ were pretreated with or without $10 \mu \mathrm{mPP} 1$ or PP3, and migration was assayed in Boyden chambers. $B$, RT-PCR for the mRNAs of nonreceptor tyrosine kinases (non-RTK) in OPCs was performed. The control $\beta$-actin is also shown. $\boldsymbol{C}$, To confirm the effects of shRNAs, RT-PCR for Fyn, Yes, or $\beta$-actin was performed using the mRNAs from OPCs infected with Fyn, Yes, or luciferase shRNA retrovirus. D, E, OPCs infected with Fyn or luciferase shRNA were incubated with or without PDGF in Boyden chambers. The number of migrating cells was counted. $F, G, O P C$ reaggregates infected with Fyn kinase or luciferase shRNA retrovirus were incubated with or without PDGF for $16 \mathrm{~h}$. The distance of migration was measured. PH, Phase-contrast images. $\boldsymbol{H}, \mathrm{OPCs}$ were infected with Yes kinase or luciferase shRNA retrovirus were incubated with or without PDGF in Boyden chambers. The number of migrating cells was counted. I, Immunoprecipitates (IP) of endogenous Fyn kinase from the lysates of OPCs were incubated with PDGF for 0-1440 min and immunoblotted with an antibody recognizing tyrosinephosphorylated residues. Total protein levels of Fyn and $\beta$-actin are also shown. The band intensity of the phosphorylated Fyn kinase was quantified. Data were evaluated using Student's $t$ test $\left({ }^{*} p<0.01\right)$.

regulation of actin-cytoskeletal-dependent cellular functions (Takenawa and Miki, 2001). Accordingly, we next asked whether Cdk5 could have the ability to phosphorylate WAVE2. Immobilized wild-type WAVE2 protein was incubated with recombinant Cdk5/p25, the activated form of Cdk5, and immunoblotted with an antibody that specifically recognizes the consensus phosphor- ylated Cdk5 target sequence. The addition of Cdk5/p25 resulted in phosphorylation of the WAVE2 protein (Fig. $6 \mathrm{~A}$, top) and also coprecipitation with WAVE2 (Fig. $6 \mathrm{~A}$, middle), indicating that Cdk5 can bind to and phosphorylate WAVE2 in vitro.

Next, to identify which domain of WAVE2 is phosphorylated by Cdk5/p25, we made plasmids encoding WAVE2 domains, 
A Time after stimulation
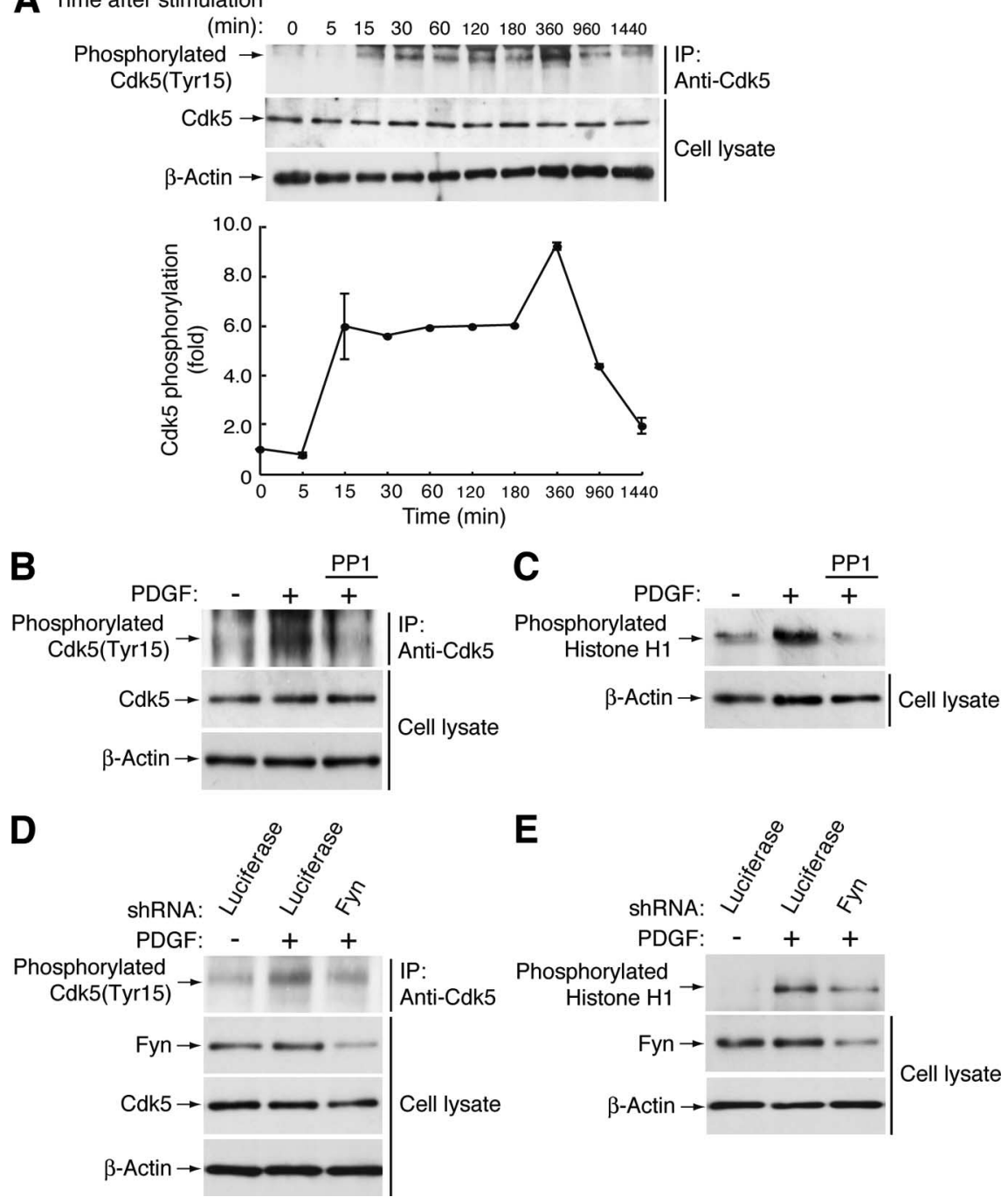

Figure 4. C $\mathrm{dk} 5$ is phosphorylated at Tyr-15 after the stimulation with PDGF. $A$, Endogenous Cdk5 proteins were immunoprecipitated from the lysates of OPCs and immunoblotted with an antibody specific for a Cdk5 phosphorylated at Tyr- 15 . Total protein levels of $\mathrm{Cdk} 5$ and $\beta$-actin are also shown. The band intensity of the phosphorylated $\mathrm{Cdk} 5$ at Tyr- 15 was quantified. $B, 0 \mathrm{PCs}$ were pretreated with or without $10 \mu \mathrm{M}$ PP1. After incubation with PDGF for $360 \mathrm{~min}$, tyrosine phosphorylation of Cdk5 was measured. Immunoblots for $\mathrm{Cdk5}$ and $\beta$-actin are also shown. C, OPCs were pretreated with or without $10 \mu \mathrm{M}$ PP1. After incubation with PDGF for $360 \mathrm{~min}$, Cdk5 activity was measured. The control $\beta$-actin is also shown. D, OPCs were infected with Fyn or luciferase shRNA retrovirus, and treated with or without PDGF stimulation. Tyrosine phosphorylation of C $\mathrm{dk} 5$ was measured. Immunoblots for Fyn kinase, $C \mathrm{dk} 5$, and $\beta$-actin are also shown. $\boldsymbol{E}, \mathrm{OPCs}$ infected with Fyn kinase or luciferase shRNA were incubated with or without PDGF and Cdk5 activity was measured. Immunoblots for Fyn kinase and $\beta$-actin are also shown. IP, Immunoprecipitate.

Thus, Cdk5 primarily phosphorylates WAVE2 at the Ser-137 position whose phosphorylation could have an effect on cellular function.

\section{Cdk5 phosphorylation of WAVE2 mediates oligodendrocyte precursor cell migration}

To clarify whether Cdk5 phosphorylates endogenous WAVE2 in OPCs after PDGF stimulation, the lysates of OPCs were immunoprecipitated with an antibody recognizing the Cdk5 target sequence and then immunoblotted with an anti-WAVE2 antibody. Stimulation with PDGF gradually increased phosphorylation of WAVE2 by Cdk5; the level of phosphorylation reached a maximum at $180 \mathrm{~min}$, having increased $\sim 9.0$-fold (Fig. 7A). Importantly, WAVE2 phosphorylation remained at an elevated level even after $24 \mathrm{~h}$. This result is also supported by a reciprocal immunoprecipitation experiment. OPC lysates were immunoprecipitated with an anti-WAVE2 antibody and then immunoblotted with an anti-phosphorylated Cdk substrate antibody. Similar to the results described above and shown in Figure $7 A$, the level of WAVE2 phosphorylation reached a maximum at 30-180 $\mathrm{min}$ and remained at a high level even after $24 \mathrm{~h}$ (supplemental Fig. 1, available at www. jneurosci.org as supplemental material). To further confirm that Fyn kinase and Cdk5 are involved in phosphorylation of WAVE2, OPCs were treated with PP1 in one experiment and roscovitine in another. PP1 and roscovitine both reduced the levels of WAVE2 phosphorylation induced by PDGF (Fig. 7B,C), indicating that both Fyn kinase and Cdk5 mediate phosphorylation of WAVE2 in OPCs.

Finally, we investigated the role of Cdk5 phosphorylation of WAVE2 at Ser137 in OPC migration. We infected OPCs with a retrovirus to manipulate expression of wild-type or Ser-137-mutated WAVE2

namely, the $\mathrm{N}$-terminal region containing the $\mathrm{SH}$ domain $(\mathrm{SH}$, amino acids 1-170), the middle region containing a proline-rich internal segment (proline, amino acids 171-284), and the C-terminal VCA domain (VCA, amino acids 285-498), and used these plasmids in an in vitro phosphorylation assay. Cdk5/p25 specifically phosphorylated $\mathrm{SH}$; it also coprecipitated with $\mathrm{SH}$ and, to a lesser extent, VCA (Fig. 6 B). Ser-137 of WAVE2 exists in the consensus Cdk5 phosphorylation sequence [S/T]PX[K/H/R] (Shelton and Johnson, 2004), which is uniquely conserved among mammalian WAVE2 proteins (Fig. 6C). To confirm whether Ser-137 is the major phosphorylation site of WAVE2, we next made a WAVE2 construct harboring the Ser-137-to-Ala mutation. As shown in Figure $6 D$ and $E$, the S137A mutation clearly impaired phosphorylation by Cdk5/p25. No difference was detected between the binding ability of Cdk5 for wild-type WAVE2 and its binding ability for the S137A mutant (Fig. 6D,E). and assessed the ability of the infected OPCs to migrate. The non-phosphorylation-mimetic S137A mutant-expressing OPCs exhibited impaired PDGF-induced migration from the aggregates (Fig. $7 D, E$ ). In contrast, the phosphomimetic mutant S137E promoted OPC migration to the level achieved by wildtype WAVE2 with PDGF stimulation (Fig. $7 D, E$ ). Importantly, this phosphomimetic mutant also promoted migration in the absence of PDGF, suggesting that phosphorylation at Ser-137 is conducive to OPC migration. Wild-type WAVE2 infection, however, had a less suppressive effect on migration (Fig. $7 D, E$ ). It is known that WAVE2 is a component of multicytoskeletal protein complex (Takenawa and Miki, 2001). Thus, exogenous WAVE2 may have a weak effect on cytoskeletal protein dynamics. Alternatively, exogenous WAVE2 may be the substrate of endogenous Cdk5 and act as a weak dominant-negative for Cdk5. In either case, it is clear that the mutation of WAVE2 at Ser-137 has an 
inhibitory effect on migration, which leads us to conclude that Cdk5 phosphorylation of WAVE2 plays a key role in PDGFinduced migration of OPCs.

\section{Discussion}

OPCs in the developing CNS are highly migratory: they disperse rapidly from their origin in the ventricular zone to their final destinations. The developmental stages of OPCs are specifically characterized by the expression of distinct transcription factors, growth factors, and cell-surface antigens. In addition, reciprocal interaction between glial and neuronal cells is strictly regulated by various growth factors and their cognate receptors. PDGF is one such regulator; it is the key controller of OPC cellular function. PDGF knock-out mice show a severe dysmyelination phenotype in all parts of the brain, but most dramatically in the optic nerves and in the cerebellum (Fruttiger et al., 1999). PDGF is secreted along the migratory pathways of OPCs (Ellison et al., 1996), and OPCs migrate toward a chemical gradient of PDGF (Armstrong et al., 1990).

Although the effect of PDGF on longdistance OPC migration has been well characterized, knowledge of the molecular mechanisms underlying OPC migration is still fragmentary. Here, we show that stimulation with PDGF promotes OPC migration through the nonreceptor tyrosine kinase Fyn, which is in turn promoted by proline-directed serine/threonine kinase Cdk5. This conclusion is supported by two findings: (1) inhibition of the cellular function of Fyn kinase or Cdk5 by a chemical inhibitor or RNA interference attenuates PDGF-induced OPC migration; (2) PDGF activates Fyn kinase to phosphorylate Cdk5 at Tyr-15. Importantly, we identify WAVE2 as the functional intracellular substrate for Cdk5. We also observe that Cdk5 directly binds to WAVE2 and phosphorylates Ser-137 within the SH domain of WAVE2 in vitro. Stimulation with PDGF in OPCs leads to WAVE2 phosphorylation in cells, whereas infection of WAVE2 harboring the Ser-137-to-Ala mutation inhibits PDGF-induced OPC migration. Together, these results demonstrate that PDGF promotes OPC migration through an unexplored mechanism, namely, a sequential phosphorylation cascade coupling Fyn kinase to Cdk5 to WAVE2.

We also detected the expression of WAVE1 and WAVE3 in primary OPCs. WAVE1-deficient mice show a dramatic decrease in the number of axons as well as prominent defects in the percentage of myelinated axons in the corpus callosum and the optic nerve (Kim HJ et al., 2006); in addition, fewer processes are observed in OLs isolated from these mice. WAVE1-deficient cells, however, are still able to migrate normally in most regions of the $\mathrm{PH}$, Phase-contrast images.
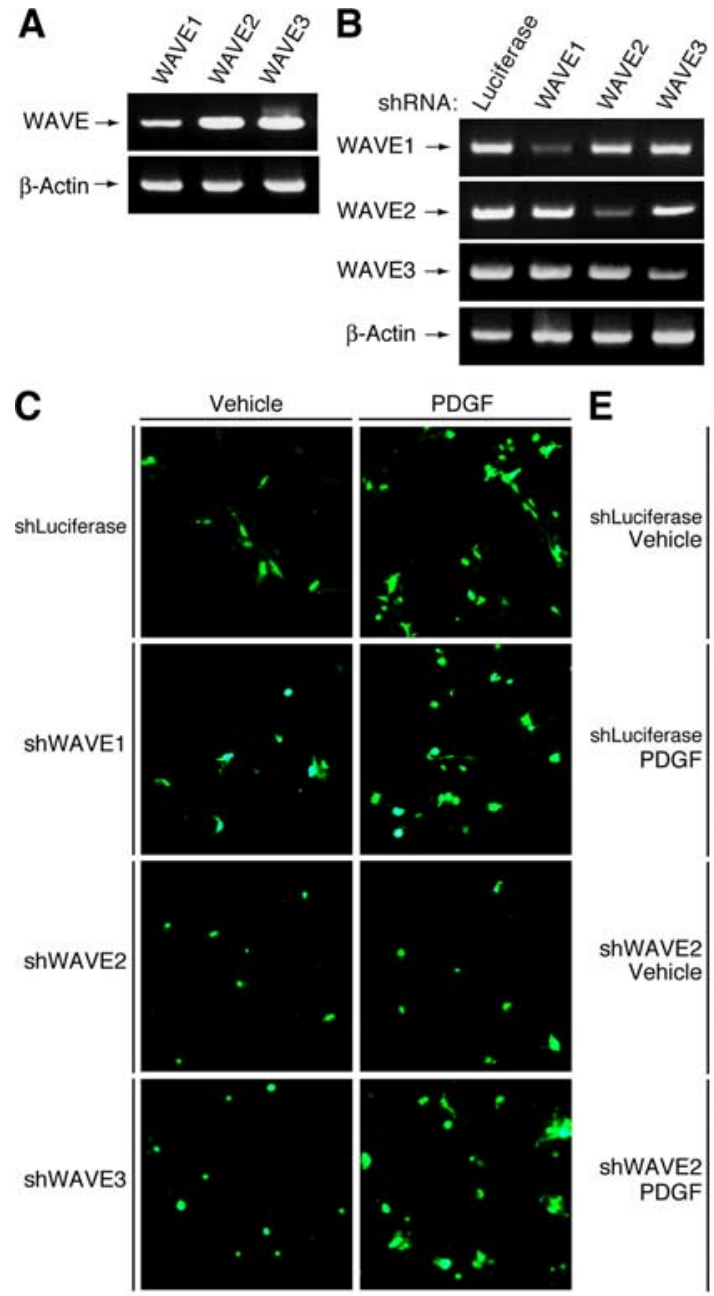

E
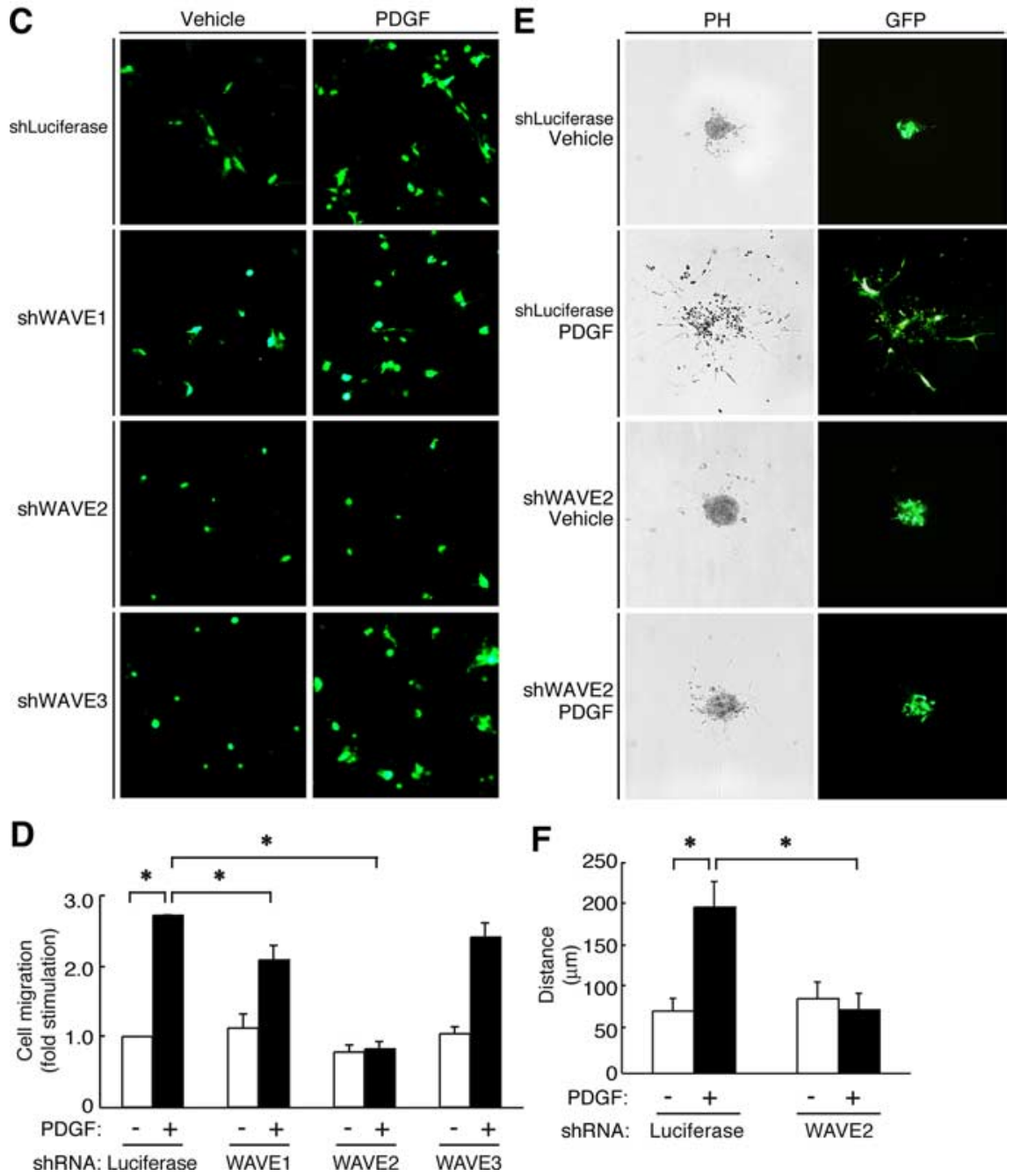

Figure 5. PDGF promotes OPC migration through WAVE2. A, RT-PCR analyses for WAVE mRNAs were performed in OPCS. The control $\beta$-actin is also shown. $\boldsymbol{B}$, To confirm the effects of shRNAs for WAVEs, RT-PCR for WAVE1, WAVE2, WAVE3, or $\beta$-actin was performed using the mRNAs from each group of infected OPCS. C, D,OPCS were infected with WAVE1, WAVE2, WAVE3, or luciferase shRNA retrovirus and incubated with or without PDGF in Boyden chambers. $\boldsymbol{E}, \boldsymbol{F}, O P C$ reaggregates infected with WAVE2 or luciferase shRNA retrovirus were incubated with or without PDGF for $16 \mathrm{~h}$. Data were evaluated using Student's $t$ test $\left({ }^{*} p<0.01\right)$.

CNS. Our study indicates that WAVE2 is the major WAVE protein required for PDGF-induced OPC migration. Further studies on the time and space regulation of WAVE family proteins will be required to clarify their complex roles in the developmental process of OLs.

Kim Y et al. (2006) reported that Cdk5 phosphorylates WAVE1 at multiple sites, resulting in an increase in dendritic spine formation in hippocampal neurons. They identified three phosphorylation sites located in a proline-rich region of WAVE1, although their sites are not conserved in WAVE2 or WAVE3. 

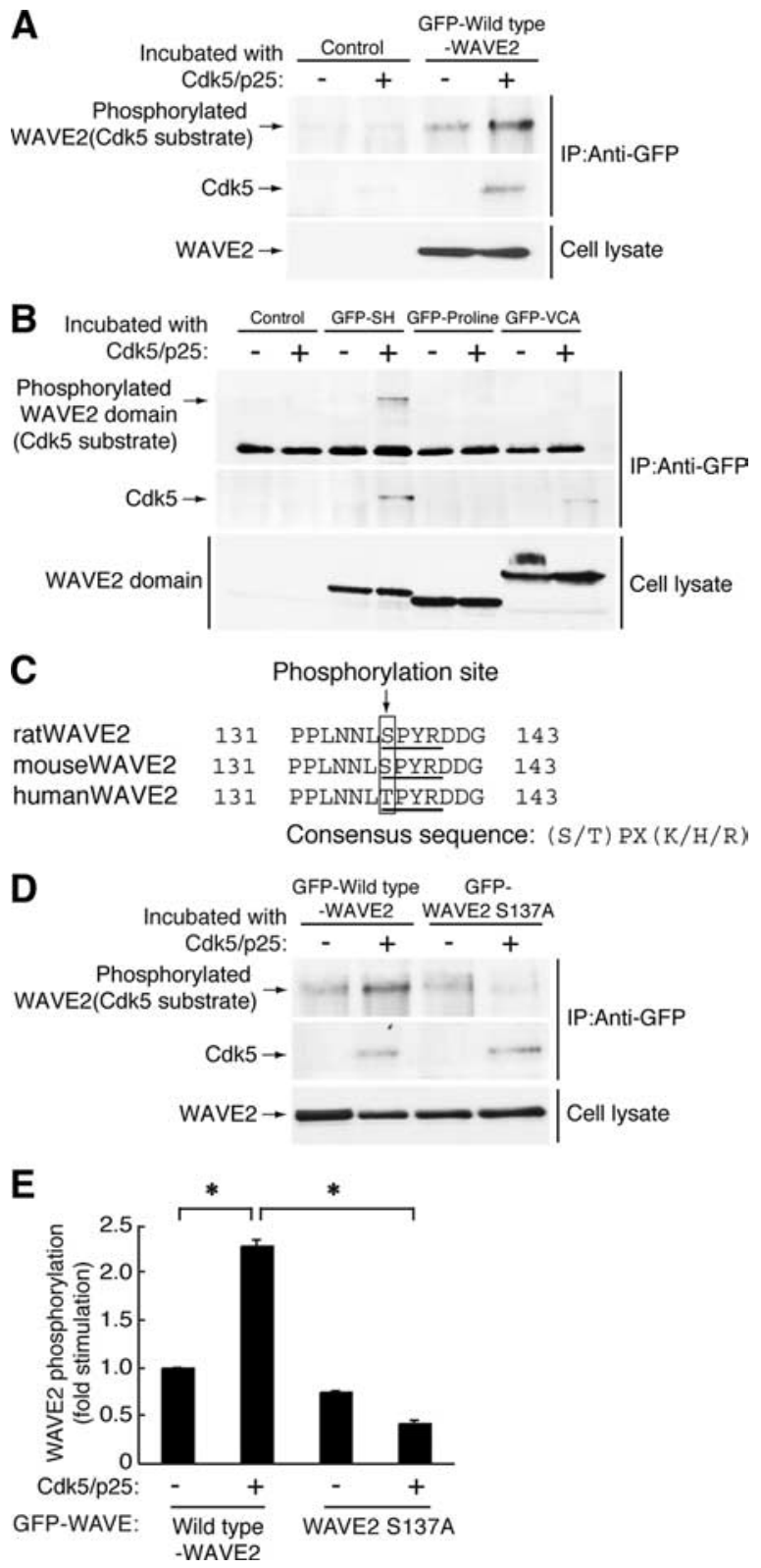

Figure 6. Cdk5 directly binds and phosphorylates WAVE2 at Ser-137. A, 293T cells were transfected with the plasmid encoding GFP-tagged wild-type WAVE2. The lysates of transfected cells were immunoprecipitated with an anti-GFP antibody. Immobilized GFP-WAVE2 protein was incubated in $30 \mu \mathrm{l}$ of reaction buffer containing $20 \mu \mathrm{m}$ cold ATP in the presence or absence of recombinant Cdk5/p25 (100 ng) for 15 min, washed, and immunoblotted with an antibody recognizing phosphorylated $\mathrm{Cdk} 5$ substrate or $\mathrm{Cdk5}$. The cell lysates were also immunoblotted with an anti-GFP antibody. B, 293T cells were transfected with the plasmid encoding GFPtagged SH, proline, or VCA of WAVE2 protein. The lysates of transfected cells were immunoprecipitated with an anti-GFP antibody, incubated with Cdk5/p25 and ATP, and immunoblotted with an antibody recognizing phosphorylated Cdk5 substrate or Cdk5. Total levels of GFPWAVE2 domains are also shown. $C$, The amino acid sequences of rat, mouse, and human WAVE2 containing a serine or tyrosine residue in the SH domain are shown. $\boldsymbol{D}$, Cells were transfected with the plasmid encoding GFP-tagged wild-type WAVE2 or WAVE2 S137A, immunoprecipitated with an anti-GFP antibody, and incubated with Cdk5/p25 and ATP. The serine phosphorylation of the constructs and their expression are also shown. $E$, Quantification of the WAVE2 phosphorylation was performed. Data were evaluated using Student's $t$ test $\left({ }^{*} p<0.01\right)$. IP, Immunoprecipitate.

Here, we identify WAVE2 as the unique substrate of Cdk5 in PDGF-induced OPC migration. Very recently, it has been reported that other proline-directed kinases, namely, extracellular signal-regulated kinase (ERK) and c-Jun N-terminal ki- nase (JNK), phosphorylate WAVE2 at a proline-rich region in response to growth factors, serum stimulation, and scratch wounding in fibroblasts (Danson et al., 2007). These phosphorylation events participate in regulating the establishment of polarity and direction of migration. Although it remains unknown whether proline-directed kinases such as ERK and JNK are involved in OPC migration, the ERK pathway, at least, is known to be the mediator of OPC proliferation and presumed to be the mediator of OPC process maturation (Stariha et al., 1997). The JNK signal, on the other hand, regulates OL survival and cell death (Zhang et al., 1996). It would be interesting to examine whether other proline-directed kinases may phosphorylate WAVE2 and possible signaling cross talk has an effect on OPC migration.

Cdk5 is expressed in all tissue; however, its kinase activity is likely to be restricted to the nervous system, because its activators, p35 and p39, are expressed solely in the nervous system (Tsai et al., 1994). We previously reported that p35 but not p39 is expressed in differentiated OLs as well (Miyamoto et al., 2007) (data not shown). Knockdown of p35 reduces the formation of the myelin weblike structures in OLs (Miyamoto et al., 2007). In this study, we have detected the expression of both p35 and p39 in OPCs. The infection of an shRNA for p39 reduces the level of OPC migration that is achieved by PDGF stimulation, but does not completely eliminate it. In addition to p39 and p35, some nonreceptor tyrosine kinases are required for Cdk5 activation in some systems. In this study, we observe that Fyn kinase plays a key role in PDGF-dependent Cdk5 activation in OPCs. In addition to Fyn kinase (Sasaki et al., 2002), c-Abl kinase has been shown to phosphorylate Tyr-15 on Cdk5 and to increase its kinase activity in neuronal cells (Zukerberg et al., 2000). Because Fyn kinase and c-Abl kinase display wide expression, it is conceivable that the phosphorylation of Cdk5 at Tyr-15 may be an important mechanism in the activation of Cdk5.

Fyn kinase also plays an important part in myelination (Umemori et al., 1994). Fyn-/- mice develop a myelin sheath that is thinner and more irregular than that found in wild-type mice. The amount of myelin basic protein in Fyn $-/-$ mice is $\sim 50 \%$ of that in wild-type mice (Sperber et al., 2001). Several studies have identified the downstream targets of Fyn kinase in OL differentiation; these substrates include not only the cytoskeletal proteins tau and $\alpha$-tubulin, but also two GTPase-activating proteins (GAPs), p190RhoGAP and p250RhoGAP, which negatively regulate the activity of the small GTPase Rho (Wolf et al., 2001; Klein et al., 2002; Taniguchi et al., 2003). Interestingly, phosphorylation of p190RhoGAP by Fyn kinase promotes its Rho-GAP activity, resulting in a decrease in Rho activity during OL differentiation. At the same time, the activation of Fyn kinase leads to upregulation of Rac and Cdc42, two other small GTPases. The mechanism by which Rho and Rac/Cdc42 are oppositely controlled is observed in many other signaling systems (Rossman et al., 2005). Generally, the balance of regulation of Rho versus $\mathrm{Rac} / \mathrm{Cdc} 42$ is achieved by multiple factors, such as GAPs and GEFs (guanine-nucleotide exchange factors), which dynamically modulate cellular morphological changes. Our studies reveal the importance of Fyn kinase in OPC migration before the onset of OL differentiation and myelination. Because migration and myelination both involve morphological changes (MelendezVasquez et al., 2004; Yamauchi et al., 2004), it is possible that Fyn kinase also regulates PDGF-dependent OPC migration through Rho GTPases.

In the present study, we identify the critical role of $\mathrm{Cdk} 5$ in PDGF-induced OPC migration. Cdk5 acts as a mediator of a 
A Time after stimulation

$(\min )$ : Phosphorylated

WAVE2(Cdk5 substrate)

$\begin{array}{llllllllll}0 & 5 & 15 & 30 & 60 & 120 & 180 & 360 & 960 & 1440\end{array}$

IP:
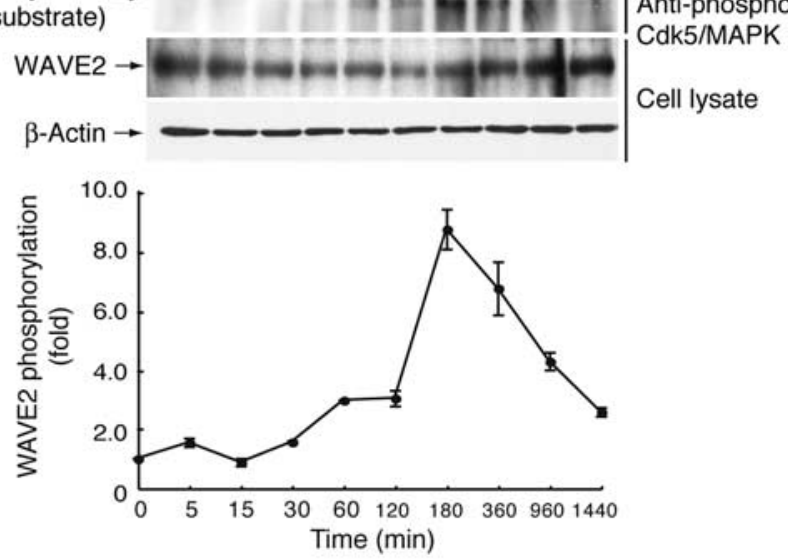

B

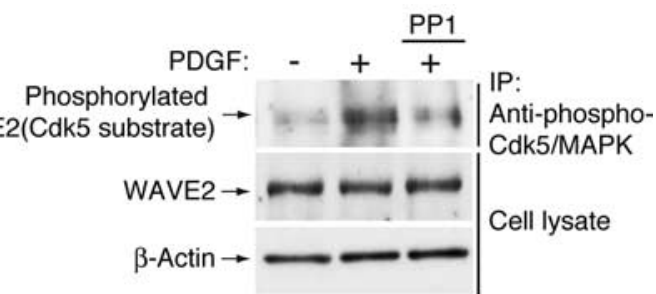

C

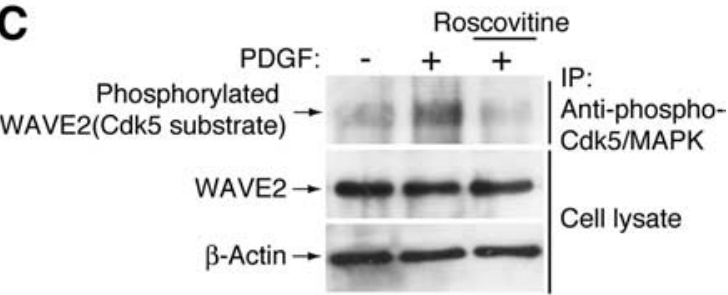

D

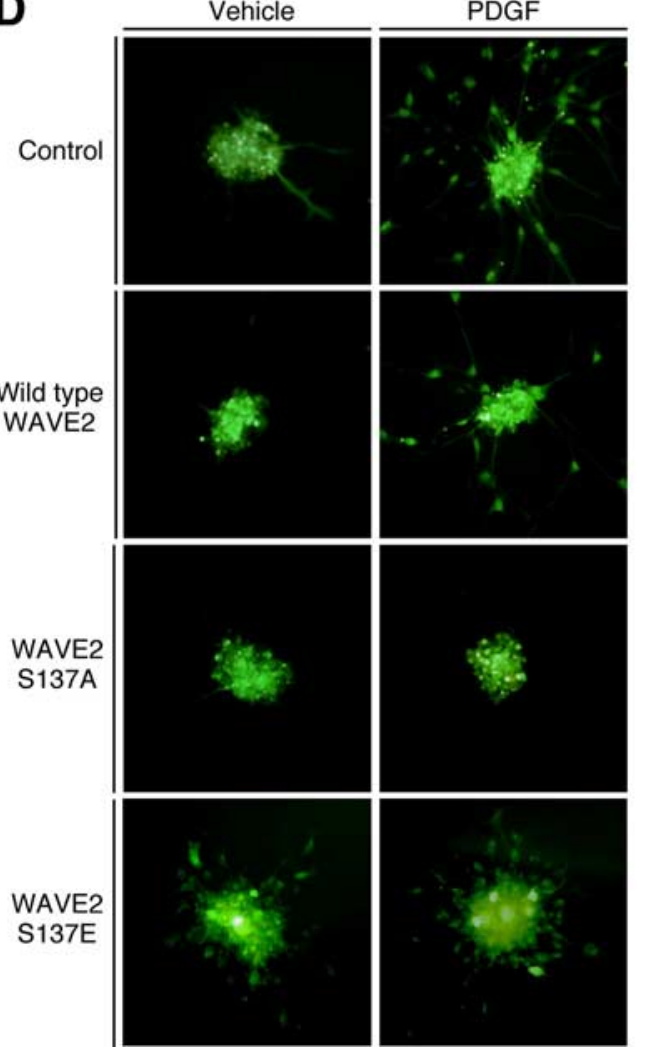

$E$
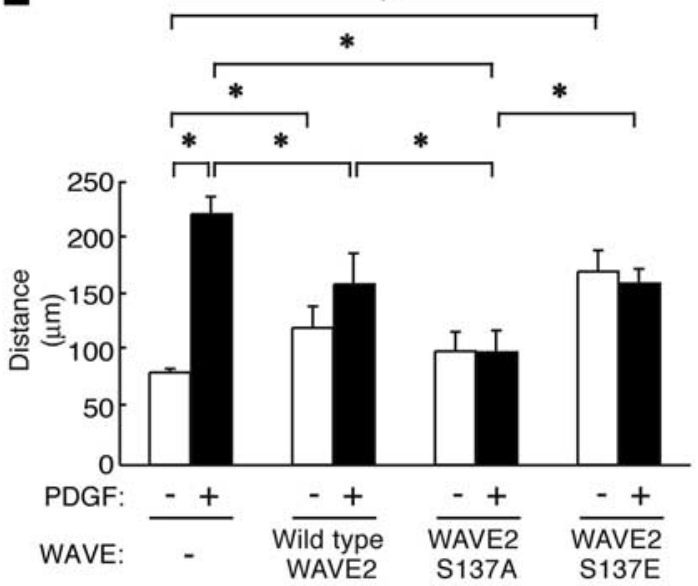

Figure 7. The importance of Ser-137 of WAVE2 in PDGF-induced migration of reaggregated OPCs. $\boldsymbol{A}$, After stimulation with PDGF for 0 -1440 min, OPCs were lysed, immunoprecipitated with an anti-phosphorylated (dk substrate antibody, and immunoblotted with an anti-WAVE2 antibody (H-110). The cell lysates were also immunoblotted with an anti-WAVE2 or $\beta$-actin antibody. $\boldsymbol{B}, \boldsymbol{C}$, OPCs were pretreated with or without $10 \mu \mathrm{M} P \mathrm{P} 1(\boldsymbol{B})$ or $10 \mu \mathrm{m}$ roscovitine $(\boldsymbol{C})$. After incubation with PDGF for 360 min, serine phosphorylation of WAVE2 was assayed. $\boldsymbol{D}, \boldsymbol{E}$, OPCs were infected with the retro-IRES, wild-type WAVE2, WAVE2 S137A, or WAVE2 S137E. At $16 \mathrm{~h}$ after stimulation with PDGF, reaggregates were fixed with paraformaldehyde, blocked, and stained with an anti-ZsGreen antibody (green). Data were evaluated using Student's $t$ test $\left(^{*} p<0.01\right)$. IP, Immunoprecipitate.

sequential cascade linking Fyn kinase to WAVE2 after PDGF stimulation. As far as we know, this is the first report on the involvement of Cdk5 in OPC migration and the first identification of WAVE2 as a functional substrate of C $\mathrm{dk} 5$. Because migration precedes myelination, certain common mechanisms may be preserved in both processes. Studies like this will thus provide not only new insights into OL biology but also possible therapeutic approaches for CNS diseases such as demyelination and damaged myelin.

\section{References}

Armstrong RC, Harvath L, Dubois-Dalcq ME (1990) Type 1 astrocytes and oligodendrocyte-type 2 astrocyte glial progenitors migrate toward distinct molecules. J Neurosci Res 27:400-407.

Barres BA, Raff MC (1999) Axonal control of oligodendrocyte development. J Cell Biol 147:1123-1128.

Bhat MA (2003) Molecular organization of axo-glial junctions. Curr Opin Neurobiol 13:552-559.

Blakemore WF, Keirstead HS (1999) The origin of remyelinating cells in the central nervous system. J Neuroimmunol 98:69-76. 
Chae T, Kwon YT, Bronson R, Dikkes P, Li E, Tsai LH (1997) Mice lacking p35, a neuronal specific activator of Cdk5, display cortical lamination defects, seizures, and adult lethality. Neuron 18:29-42.

Dahl JP, Wang-Dunlop J, Gonzales C, Goad MEP, Mark RJ, Kwak SP (2003) Characterization of the WAVE1 knock-out mouse: implications for CNS development. J Neurosci 23:3343-3352.

Danson CM, Pocha SM, Bloomberg GB, Cory GO (2007) Phosphorylation of WAVE2 by MAP kinases regulates persistent cell migration and polarity. J Cell Sci 120:4144-4154.

Ellison JA, Scully SA, de Vellis J (1996) Evidence for neuronal regulation of oligodendrocyte development: cellular localization of platelet-derived growth factor alpha receptor and A-chain mRNA during cerebral cortex development in the rat. J Neurosci Res 45:28-39.

Fruttiger M, Karlsson L, Hall AC, Abramsson A, Calver AR, Boström H, Willetts K, Bertold CH, Heath JK, Betsholtz C, Richardson WD (1999) Defective oligodendrocyte development and severe hypomyelination in PDGF-A knockout mice. Development 126:457-467.

Heldin CH, Westermark B (1989) Platelet-derived growth factor. Three isoforms and two receptor types. Trends Genet 5:108-111.

Kim HJ, DiBernardo AB, Sloane JA, Rasband MN, Solomon D, Kosaras B, Kwak SP, Vartanian TK (2006) WAVE1 is required for oligodendrocyte morphogenesis and normal CNS myelination. J Neurosci 26:5849-5859.

Kim Y, Sung JY, Ceglia I, Lee KW, Ahn JH, Halford JM, Kim AM, Kwak SP, Park JB, Ho Ryu S, Schenck A, Bardoni B, Scott JD, Nairn AC, Greengard P (2006) Phosphorylation of WAVE1 regulates actin polymerization and dendritic spine morphology. Nature 442:814-817.

Klein C, Kramer EM, Cardine AM, Schraven B, Brandt R, Trotter J (2002) Process outgrowth of oligodendrocytes is promoted by interaction of fyn kinase with the cytoskeletal protein tau. J Neurosci 22:698-707.

Ko J, Humbert S, Bronson RT, Takahashi S, Kulkarni AB, Li E, Tsai LH (2001) p35 and p39 are essential for cyclin-dependent kinase 5 function during neurodevelopment. J Neurosci 21:6758-6771.

Lee JC, Mayer-Proschel M, Rao MS (2000) Gliogenesis in the central nervous system. Glia 30:105-121.

McKinnon RD, Matsui T, Dubois-Dalcq M, Aaronson SA (1990) FGF modulates the PDGF-driven pathway of oligodendrocyte development. Neuron 5:603-614.

Melendez-Vasquez CV, Einheber S, Salzer JL (2004) Rho kinase regulates Schwann cell myelination and formation of associated axonal domains. J Neurosci 24:3953-3963.

Miki H, Suetsugu S, Takenawa T (1998) WAVE, a novel WASP-family protein involved in actin reorganization induced by Rac. EMBO J 17:6932-6941.

Miller RH (2002) Regulation of oligodendrocyte development in the vertebrate CNS. Prog Neurobiol 67:451-467.

Miyamoto Y, Yamauchi J, Tanoue A, Wu C, Mobley WC (2006) TrkB binds and tyrosine-phosphorylates Tiaml leading to activation of Racl and induction of changes in cellular morphology. Proc Natl Acad Sci U S A 103:10444-10449.

Miyamoto Y, Yamauchi J, Chan JR, Okada A, Tomooka Y, Hisanaga S, Tanoue A (2007) Cdk5 regulates differentiation of oligodendrocyte precursor cells through the direct phosphorylation of paxillin. J Cell Sci 120:4355-4366.

Mudhar HS, Pollock RA, Wang C, Stiles CD, Richardson WD (1993) PDGF and its receptor in the developing rodent retina and optic nerve. Development 118:539-552.

Noble M, Murray K, Stroobant P, Waterfield MD, Riddle P (1988) Plateletderived growth factor promotes division and motility and inhibits premature differentiation of the oligodendrocyte/type-2 astrocyte progenitor cell. Nature 333:560-562.

Ohshima T, Ward JM, Huh CG, Longenecker G, Veeranna, Pant HC, Brady RO, Martin LJ, Kulkarni AB (1996) Targeted disruption of cyclindependent kinase 5 gene results in abnormal corticogenesis, neuronal pathology, and perinatal death. Proc Natl Acad Sci USA 93:11173-11178.

Osterhout DJ, Wolven A, Wolf RM, Resh MD, Chao MV (1999) Morpho- logical differentiation of oligodendrocytes requires activation of Fyn tyrosine kinase. J Cell Biol 145:1209-1218.

Pringle N, Collarini EJ, Mosley MJ, Heldin CH, Westermark B, Richardson WD (1989) PDGF A chain homodimers drive proliferation of bipotential (O-2A) glial progenitor cells in the developing rat optic nerve. EMBO J 8:1049-1056.

Richardson WD, Pringle N, Mosley MJ, Westermark B, Dubois-Dalcq M (1988) A role for platelet-derived growth factor in normal gliogenesis in the central nervous system. Cell 53:309-319.

Rossman KL, Der CJ, Sondek J (2005) GEF means go: turning on RHO GTPases with guanine nucleotide-exchange factors. Nat Rev Mol Cell Biol 6:167-180.

Sasaki Y, Cheng C, Uchida Y, Nakajima O, Ohshima T, Yagi T, Taniguchi M, Nakayama T, Kishida R, Kudo Y, Ohno S, Nakamura F, Goshima Y (2002) Fyn and Cdk5 mediate Semaphorin-3A signaling, which is involved in regulation of dendrite orientation in cerebral cortex. Neuron 35:907-920.

Shelton SB, Johnson GVW (2004) Cyclin-dependent kinase-5 in neurogeneration. J Neurochem 88:1313-1326.

Sperber BR, Boyle-Walsh EA, Engleka MJ, Gadue P, Peterson AC, Stein PL, Scherer SS, McMorris FA (2001) A unique role for Fyn in CNS myelination. J Neurosci 21:2039-2047.

Stariha RL, Kikuchi S, Siow YL, Pelech SL, Kim M, Kim SU (1997) Role of extracellular signal-regulated protein kinase 1 and 2 in oligodendroglial process extension. J Neurochem 68:945-953.

Symons M, Derry JM, Karlak B, Jiang S, Lemahieu V, Mccormick F, Francke U, Abo A (1996) Wiskott-Aldrich syndrome protein, a novel effector for the GTPase CDC42Hs, is implicated in actin polymerization. Cell 84:723-734.

Takenawa T, Miki H (2001) WASP and WAVE family proteins; key molecules for rapid rearrangement of cortical actin filaments and cell movement. J. Cell Sci 114:1801-1809.

Tang D, Yeung J, Lee KY, Matsushita M, Matsui H, Tomizawa K, Hatase O, Wang JH (1995) An isoform of the neuronal cyclin-dependent kinase 5(Cdk5) activator. J Biol Chem 270:26897-26903.

Taniguchi S, Liu H, Nakazawa T, Yokoyama K, Tezuka T, Yamamoto T (2003) p250GAP, a neural RhoGAP protein, is associated with and phosphorylated by Fyn. Biochem Biophys Res Commun 306:151-155.

Thomas SM, Brugge JS (1997) Cellular functions regulated by Src family kinases. Annu Rev Cell Dev Biol 13:513-609.

Tsai LH, Delalle I, Caviness VS Jr, Chae T, Harlow E (1994) p35 is a neuralspecific regulatory subunit of cyclin-dependent kinase 5. Nature 371:419-423.

Umemori H, Sato S, Yagi T, Aizawa S, Yamamoto T (1994) Initial events of myelination involve Fyn tyrosine kinase signaling. Nature 367:572-576.

Wolf RM, Wilkes JJ, Chao MV, Resh MD (2001) Tyrosine phosphorylation of p190RhoGAP by Fyn regulates oligodendrocyte differentiation. J Neurobiol 49:62-78.

Yamauchi J, Chan JR, Shooter EM (2004) Neurotrophins regulate Schwann cell migration by activating divergent signaling pathways dependent on Rho GTPases. Proc Natl Acad Sci U S A 101:8774-8779.

Yamazaki D, Suetsugu S, Miki H, Kataoka Y, Nishikawa S, Fujiwara T, Yoshida N, Takenawa T (2003) WAVE2 is required for directed cell migration and cardiovascular development. Nature 424:452-456.

Yeh HJ, Ruit KG, Wang YX, Parks WC, Snider WD, Deuel TF (1991) PDGF A-chain gene is expressed by mammalian neurons during development and in maturity. Cell 64:209-216.

Zhang P, Miller BS, Rosenzweig SA, Bhat NR (1996) Activation of c-jun N-terminal kinase/stress-activated protein kinase in primary glial cultures. J Neurosci Res 46:114-121.

Zhang SC, Ge B, Duncan ID (1999) Adult brain retains the potential to generate oligodendroglial progenitors with extensive myelination capacity. Proc Natl Acad Sci U S A 96:4089-4094.

Zukerberg LR, Patrick GN, Nikolic M, Humbert S, Wu CL, Lanier LM, Gertler FB, Vidal M, Van Etten RA, Tsai LH (2000) Cables links Cdk5 and $\mathrm{c}-\mathrm{Abl}$ and facilitates $\mathrm{Cdk} 5$ tyrosine phosphorylation, kinase upregulation, and neurite outgrowth. Neuron 26:633-646. 